\title{
RATES OF EIGENVALUES ON A DUMBBELL DOMAIN. SIMPLE EIGENVALUE CASE
}

\author{
JOSE M. ARRIETA
}

\begin{abstract}
We obtain the first term in the asymptotic expansion of the eigenvalues of the Laplace operator in a typical dumbbell domain in $\mathbb{R}^{2}$. This domain consists of two disjoint domains $\Omega^{L}, \Omega^{R}$ joined by a channel $R_{\varepsilon}$ of height of the order of the parameter $\varepsilon$. When an eigenvalue approaches an eigenvalue of the Laplacian in $\Omega^{L} \cup \Omega^{R}$, the order of convergence is $\varepsilon$, while if the eigenvalue approaches an eigenvalue which comes from the channel, the order is weaker: $\varepsilon|\ln \varepsilon|$. We also obtain estimates on the behavior of the eigenfunctions.
\end{abstract}

\section{INTRODUCTION}

In this paper we deal with the problem of the behavior of the eigenvalues and eigenfunctions of the Laplace operator with Neumann boundary condition when the domain is singularly perturbed.

The prototype of perturbation of the domain we are interested in is the socalled dumbbell domain which consists of two disjoint domains $\Omega^{L}$ and $\Omega^{R}$ joined by a thin channel $R_{\varepsilon}$ which approaches a line segment as the parameter $\varepsilon$ approaches zero. To fix the ideas let us consider that we work in $\mathbb{R}^{2}$ and the channel is given by $R_{\varepsilon}=\{(x, y): 0<x<1,0<y<\varepsilon g(x)\}$ where $g \in C^{1}(0,1)$ and $g(x) \geq 0$ for all $x \in[0,1]$ (see Figure 1).

The basic fact about the behavior of the eigenvalues for this kind of perturbation is the following: if $\left\{\mu_{k}\right\}_{k=1}^{\infty}$ are the eigenvalues of $-\Delta$ in $\Omega \equiv \Omega^{L} \cup \Omega^{R}$ with Neumann B.C., if $\left\{\tau_{j}\right\}_{j=1}^{\infty}$ are the eigenvalues of the operator $-\frac{1}{g}\left(g u_{x}\right)_{x}$ in $(0,1)$ with Dirichlet B.C. and if we denote by $\left\{\lambda_{n}\right\}_{n=1}^{\infty}=\left\{\mu_{k}\right\}_{k=1}^{\infty} \cup\left\{\tau_{j}\right\}_{j=1}^{\infty}$, ordered and counting the multiplicity, then if $\left\{\lambda_{n}^{\varepsilon}\right\}_{n=1}^{\infty}$ are the eigenvalues of $-\Delta$ in $\Omega_{\varepsilon}=\Omega^{L} \cup R_{\varepsilon} \cup \Omega^{R}$, with Neumann B.C., we have, $\lambda_{n}^{\varepsilon} \stackrel{\varepsilon \rightarrow 0}{\longrightarrow} \lambda_{n}$.

This result gives us a characterization of the eigenvalue behavior under this kind of perturbation and allows us to sketch a bifurcation diagram of $\lambda_{n}^{\varepsilon}$ with respect to the parameter $\varepsilon$ (see Figure 2).

Notice that the eigenvalues of $-\frac{1}{g}\left(g u_{x}\right)_{x}$ in $(0,1)$, with a Dirichlet B.C., play a role since they are the limit of the eigenvalues of $-\Delta$ in $R_{\varepsilon}$, with a Dirichlet B.C. in $\Gamma_{\varepsilon}=\partial R_{\varepsilon} \cap \partial \Omega$ and Neumann everywhere else (see [11]).

Received by the editors February 8, 1994 and, in revised form, October 6, 1994.

1991 Mathematics Subject Classification. Primary 35P15. 


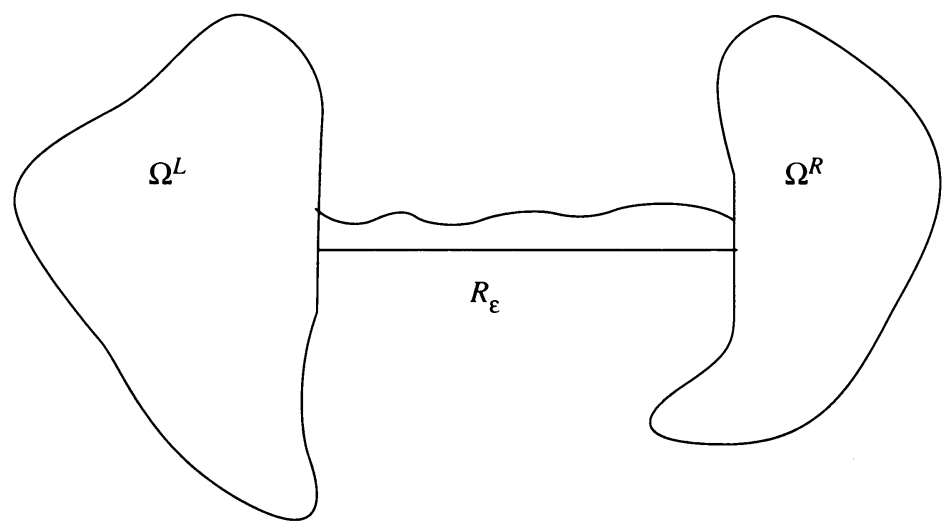

Figure 1

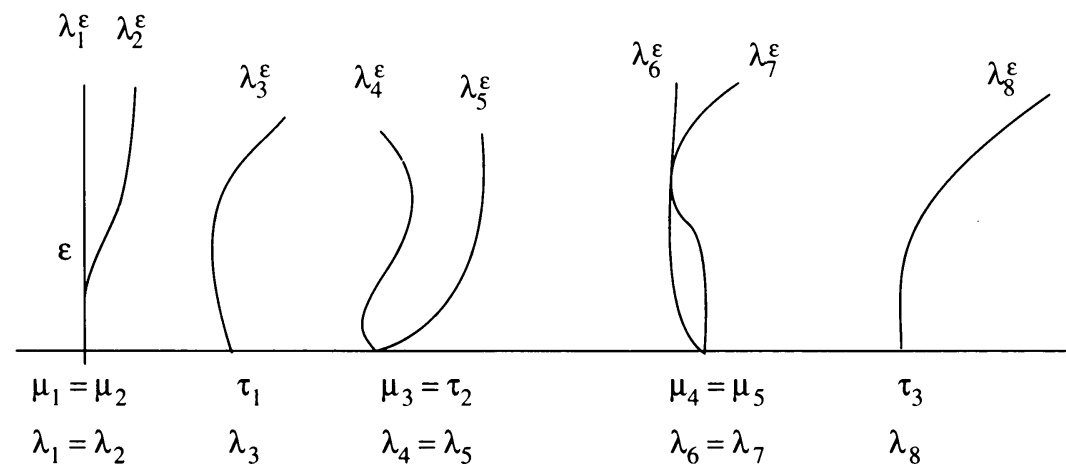

FIGURE 2

The case $g \equiv 1$ and dimension greater than or equal 3 was studied by Jimbo in [15]. He also obtained nice estimates on the $L^{\infty}$ behavior of the eigenfunctions. The work in [13] includes results in this case also.

For the case $g \not \equiv 1$, see the work in [17], where they obtained this kind of result even for an $N$-dumbbell domain, that is, a family of $N$ pairwise disjoint domains joined by several thin channels.

In a much more general setting, including the case where the channel presents a high oscillating boundary $\left(R_{\varepsilon}=\left\{(x, y): 0<x<1,0<y<\varepsilon g\left(x \varepsilon^{-\alpha}\right)\right\}\right.$ with $0<\alpha<1)$, or even, in dimension $d \geq 3$, where the channel approaches a manifold of dimension $d-k$ for $k=1, \ldots, d-1$, this result was studied by the author in [1], [2].

Many other authors have studied similar kinds of singular perturbations of the domain in different situations; see [5], [6], [7], [14], [18], [22], [12], [11].

For other kinds of singular perturbations the reader is referred to [3], [21], [19], [20].

It is clear that from the fact that $\lambda_{n}^{\varepsilon} \stackrel{\varepsilon \rightarrow 0}{\longrightarrow} \lambda_{n}$ we simply obtain the continuity at $\varepsilon=0$ of the branches depicted in Figure 2, and in order to draw a more accurate bifurcation diagram we will need to analyse the asymptotic behavior as $\varepsilon \rightarrow 0$ of the quantities $\lambda_{n}^{\varepsilon}-\lambda_{n}$. In this direction there are some results. 
Jimbo and Morita in [17] treated the case of an $N$-dumbbell domain. In this case it it clear that $\lambda_{i}^{\varepsilon} \stackrel{\varepsilon \rightarrow 0}{\longrightarrow} 0$ for $i=1, \ldots, N$, while $\lambda_{N+1}^{\varepsilon}$ is uniformly bounded away from zero. They were able to prove that $\lambda_{i}^{\varepsilon}=C_{i} \varepsilon^{d-1}+o\left(\varepsilon^{d-1}\right)$, for $i=1, \ldots, N$, where $d$ is the dimension of the ambient space, and $C_{i}$ are certain constants which depend on the geometry of the domain.

Jimbo in [16] is able to obtain the first term in the asymptotic expansion of $\lambda_{n}^{\varepsilon}$ for a typical dumbbell domain, in dimension $d \geq 3$, where the channel is straight, that is, $g \equiv 1$, and with the essential condition that $\left\{\mu_{k}\right\} \cap\left\{\tau_{j}\right\}=\varnothing$ and $\lambda_{n}^{\varepsilon}$ approaches an element of $\left\{\mu_{k}\right\}$. He obtains that $\lambda_{n}^{\varepsilon}=\mu_{k}+C_{n} \varepsilon^{d-1}+$ $o\left(\varepsilon^{d-1}\right)$. In this work nothing is said about the case when $\lambda_{n}^{\varepsilon}$ approaches an element of $\left\{\tau_{j}\right\}$.

In [6], the authors find upper bounds for $\left|\lambda_{n}^{\varepsilon}-\lambda_{n}\right|$, obtaining that

(i) If $\lambda_{n} \in\left\{\mu_{k}\right\} \backslash\left\{\tau_{j}\right\}$,

$$
\begin{array}{ll}
\left|\lambda_{n}^{\varepsilon}-\lambda_{n}\right| \leq C|\ln \varepsilon|^{-1 / 2}, & \text { dimension }=2, \\
\left|\lambda_{n}^{\varepsilon}-\lambda_{n}\right| \leq C \varepsilon^{(d-2) / d}, & \text { dimension }=d \geq 3 .
\end{array}
$$

(ii) If $\lambda_{n} \in\left\{\tau_{j}\right\} \backslash\left\{\mu_{k}\right\}$,

$$
\begin{array}{ll}
\left|\lambda_{n}^{\varepsilon}-\lambda_{n}\right| \leq C \varepsilon^{1 / 2}|\ln \varepsilon|, & \text { dimension }=2, \\
\left|\lambda_{n}^{\varepsilon}-\lambda_{n}\right| \leq C \varepsilon^{1 / 2}, & \text { dimension }=d \geq 2 .
\end{array}
$$

In this paper we will work with a typical dumbbell domain in $\mathbb{R}^{2}$ with a general $g \in C^{1}(0,1)$. We will consider a $\lambda_{n}$ with the condition that $\lambda_{n-1}<\lambda_{n}<$ $\lambda_{n+1}$ and we will be able to obtain the first term in the asymptotic expansion of $\lambda_{n}^{\varepsilon}-\lambda_{n}$, as $\varepsilon \rightarrow 0$, for both situations: (a) $\lambda_{n}=\mu_{k}$ and (b) $\lambda_{n}=\tau_{k}$. Actually, we will obtain the constants $A_{k}$ and $B_{k}$, such that

(a) If $\lambda_{n}=\mu_{k}$ for certain $k$, then

$$
\lambda_{n}^{\varepsilon}=\mu_{k}+A_{k} \varepsilon+o(\varepsilon)
$$

(b) If $\lambda_{n}=\tau_{k}$ for certain $k$, then $B_{k}<0$, and

$$
\lambda_{n}^{\varepsilon}=\tau_{k}+B_{k} \varepsilon|\ln \varepsilon|+o(\varepsilon|\ln \varepsilon|) .
$$

Notice that the rates obtained for (a) and (b) are different.

Case (a) is in accordance with the result from [16], which was obtained for dimension $d \geq 3$ and with $g \equiv 1$.

Case (b) is new and as far as the author knows it has never been established, not even for the case $g \equiv 1$. We can also see the upper bounds obtained by [6] are not optimal.

With this result now we can draw a more accurate bifurcation diagram (see Figure 3). 

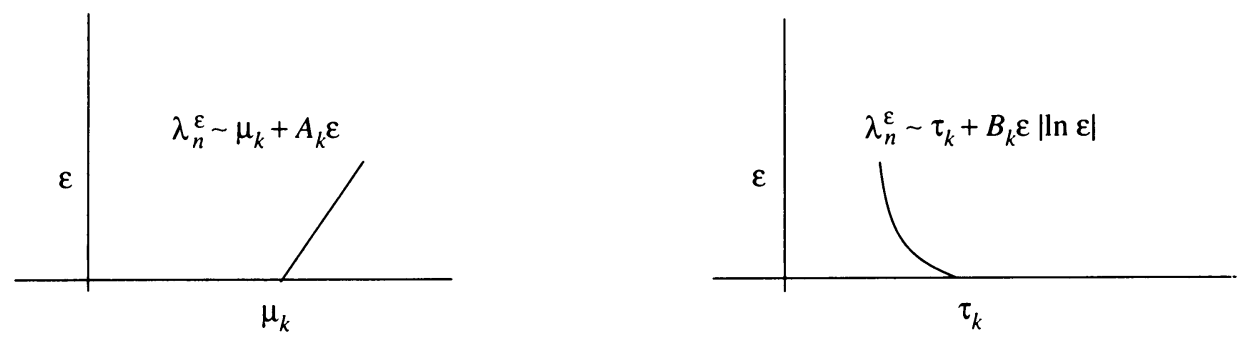

FIgURE 3

With respect to the case of a multiple eigenvalue, we will just mention that, for the case where $d \geq 3, g \equiv 1, \lambda_{r-1}<\lambda_{r}=\cdots=\lambda_{s}<\lambda_{s+1}$ and $\lambda_{r}, \ldots, \lambda_{s} \notin$ $\left\{\tau_{k}\right\}$, the rates were obtained in [16]. But, for example, in the case where $\lambda_{n-1}<\lambda_{n}=\lambda_{n+1}<\lambda_{n+2}$ and $\lambda_{n}=\mu_{k}, \lambda_{n+1}=\tau_{j}$ for certain $k$ and $j$, the asymptotic expansion is essentially different from the one obtained in case (a) or (b). This will be treated in a subsequent paper.

\section{NotATION AND MAIN RESULTS}

In this section we make precise the notation and the results stated in the introductory section.

We will consider a fixed smooth and bounded open set $\Omega \subset \mathbb{R}^{2}$, with the property that there exists an $l>0$ such that

$$
\begin{gathered}
\Omega \cap\left\{(x, y): x^{2}+y^{2}<l^{2}\right\}=\left\{(x, y): x^{2}+y^{2}<l^{2}, x<0\right\}, \\
\Omega \cap\left\{(x, y):(x-1)^{2}+y^{2}<l^{2}\right\}=\left\{(x, y):(x-1)^{2}+y^{2}<l^{2}, x>1\right\}, \\
\Omega \cap\{(x, y): 0<x<1,-l<y<l\}=\varnothing,
\end{gathered}
$$

so that $\{(0, y):-l<y<l\} \cup\{(1, y):-l<y<l\} \subset \partial \Omega$. Notice that we do not specify any connectedness property on $\Omega$. Therefore we could have the situation from Figure 1 or even the one from Figure 4.

Let $B \subset \partial \Omega$ be a regular part of the boundary of $\Omega$, possibly empty, with the property that $B \cap(\{(0, y):-l<y<l\} \cup\{(1, y):-l<y<l\})=\varnothing$. We will consider the following eigenvalue problem:

$$
\begin{aligned}
-\Delta \varphi & =\mu \varphi, & & \Omega, \\
\varphi & =0, & & B, \\
\frac{\partial \varphi}{\partial n} & =0, & & \partial \Omega \backslash B,
\end{aligned}
$$

and we will denote by $\left\{\mu_{n}\right\}_{n=1}^{\infty}$ the set of eigenvalues of (2.1) ordered and counting multiplicity, and by $\left\{\phi_{n}\right\}_{n=1}^{\infty}$ the corresponding set of orthonormalized eigenfunctions.

Let $g \in C^{1}(0,1)$, and define $R_{\varepsilon}=\{(x, y): 0<x<1,0<y<\varepsilon g(x)\} \subset$ $\mathbb{R}^{2}, \Gamma_{\varepsilon}^{L}=\{(0, y): 0<y<\varepsilon g(0)\}, \Gamma_{\varepsilon}^{R}=\{(1, y): 0<y<\varepsilon g(1)\}$ where $\varepsilon \in\left(0, \varepsilon_{0}\right)$ for some small $\varepsilon_{0}$. Denote also $\Gamma_{\varepsilon}=\Gamma_{\varepsilon}^{L} \cup \Gamma_{\varepsilon}^{R}$ and consider the eigenvalue problem

$$
\begin{aligned}
-\Delta \gamma & =\tau \gamma, & & R_{\varepsilon}, \\
\varphi & =0, & & \Gamma \varepsilon, \\
\frac{\partial \varphi}{\partial n} & =0, & & \partial R_{\varepsilon} \backslash \Gamma_{\varepsilon} .
\end{aligned}
$$




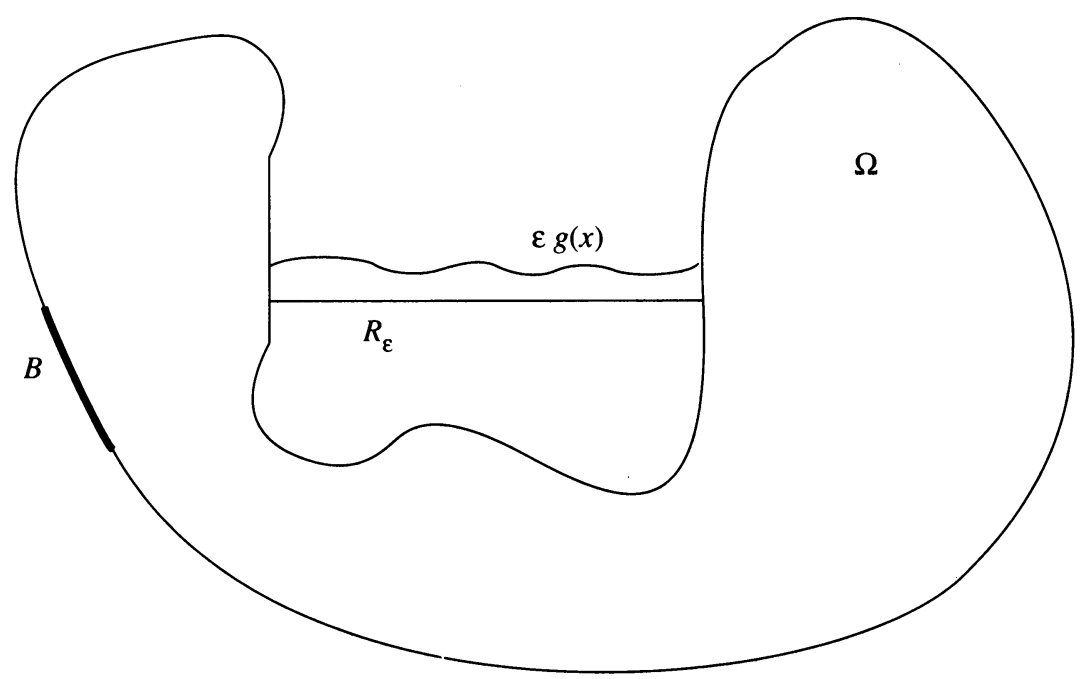

FIGURE 4

Denote by $\left\{\tau_{n}^{\varepsilon}\right\}_{n=1}^{\infty}$ the set of eigenvalues of (2.2) ordered and counting multiplicity and by $\left\{\gamma_{n}^{\varepsilon}\right\}_{n=1}^{\infty}$ the corresponding set of orthonormalized eigenfunctions.

Let $\left\{\left(\tau_{n}, \gamma_{n}\right)\right\}$ be the eigenpairs of the problem

$$
\begin{aligned}
-\frac{1}{g}\left(g \gamma_{x}\right)_{x} & =\tau \gamma, \quad x \in(0,1), \\
\gamma(0) & =0, \quad \gamma(1)=0 .
\end{aligned}
$$

It is well known that the following result holds:

Theorem 2.1. For all $n \in N$, we have

$$
\tau_{n}^{\varepsilon}-\tau_{n}=O\left(\varepsilon^{2}\right), \quad\left\|\gamma_{n}^{\varepsilon}-\left(\gamma_{n}^{\varepsilon}, \varepsilon^{-1 / 2} \gamma_{n}\right)_{L^{2}\left(R_{\varepsilon}\right)} \varepsilon^{-1 / 2} \gamma_{n}\right\|_{H^{1}\left(R_{\varepsilon}\right)}^{2}=O\left(\varepsilon^{2}\right) .
$$

For the proof of this result see [11] or [1].

Define the domain $\Omega_{\varepsilon}=\Omega \cup \Gamma_{\varepsilon} \cup R_{\varepsilon}$. Consider the eigenvalue problem:

$$
\begin{aligned}
-\Delta \varphi & =\lambda \varphi, & & \Omega_{\varepsilon}, \\
\varphi & =0, & & B, \\
\frac{\partial \varphi}{\partial n} & =0, & & \partial \Omega_{\varepsilon} \backslash B,
\end{aligned}
$$

and denote by $\left\{\lambda_{n}^{\varepsilon}\right\}_{n=1}^{\infty}$ the set of eigenvalues of (2.4) and by $\left\{\varphi_{n}^{\varepsilon}\right\}_{n=1}^{\infty}$ the corresponding set of orthonormalized eigenfunctions.

Define

$$
\left\{\lambda_{n}\right\}_{n=1}^{\infty}=\left\{\mu_{i}\right\}_{i=1}^{\infty} \cup\left\{\tau_{n}\right\}_{n=1}^{\infty},
$$

always ordered and counting the multiplicity.

Let $E$ be a bounded, linear extension operator from $H^{1}(\Omega)$ to $H^{1}\left(\mathbb{R}^{2}\right)$, and define the functions

$$
\begin{aligned}
& \psi_{n}^{\varepsilon}=E \phi_{k}, \quad \text { if } \lambda_{n}=\mu_{k}, \\
& \psi_{n}^{\varepsilon}=\left\{\begin{array}{ll}
0, & \Omega \\
\varepsilon^{-1 / 2} \gamma_{k}, & R_{\varepsilon}
\end{array} \text { if } \lambda_{n}=\tau_{k} .\right.
\end{aligned}
$$

The following result, in a more general setting, can be found in [1], [2]. 
Theorem 2.2. For all $n \in N$ we have

$$
\lambda_{n}^{\varepsilon}=\lambda_{n}+o(1) \text {. }
$$

Moreover, if $\lambda_{r-1}<\lambda_{r}=\lambda_{r+1}=\cdots=\lambda_{s}<\lambda_{s+1}$, then

$$
\left\|\varphi_{n}^{\varepsilon}-\sum_{i=r}^{s}\left(\varphi_{n}^{\varepsilon}, \psi_{i}^{\varepsilon}\right)_{\Omega_{\varepsilon}} \psi_{i}^{\varepsilon}\right\|_{H^{1}\left(\Omega_{\varepsilon}\right)} \stackrel{\varepsilon \rightarrow 0}{\longrightarrow} 0, \quad n=r, r+1, \ldots, s .
$$

Corollary 2.3. For all $n \in N$ we have $\left\|\partial \varphi_{n}^{\varepsilon} / \partial y\right\|_{R_{\varepsilon}} \stackrel{\varepsilon \rightarrow 0}{\longrightarrow} 0$.

Remark 2.4. Notice that in the case where $\lambda_{n-1}<\lambda_{n}<\lambda_{n+1}$, statement (2.7) tells us that $\lambda_{n}^{\varepsilon}$ is a simple eigenvalue for $\varepsilon$ small enough, and therefore $\varphi_{n}^{\varepsilon}$ is unique up to a change of sign. Moreover, (2.8) can be written as

$$
\left\|\varphi_{n}^{\varepsilon}-\left(\varphi_{n}^{\varepsilon}, \psi_{n}^{\varepsilon}\right)_{\Omega_{\varepsilon}} \psi_{n}^{\varepsilon}\right\|_{H^{1}\left(\Omega_{\varepsilon}\right)} \stackrel{\varepsilon \rightarrow 0}{\longrightarrow} 0,
$$

and this implies $\left(\varphi_{n}^{\varepsilon}, \psi_{n}^{\varepsilon}\right)_{\Omega_{\varepsilon}}^{2}=1+o(1)$. From now on we will always choose $\varphi_{n}^{\varepsilon}$ with the following sign criterion:

$$
\left(\varphi_{n}^{\varepsilon}, \psi_{n}^{\varepsilon}\right)_{\Omega_{\varepsilon}}=1+o(1) .
$$

Therefore, we will always have

(a) If $\lambda_{n}=\mu_{k} ;\left\|\varphi_{n}^{\varepsilon}-\phi_{k}\right\|_{H^{1}(\Omega)} \stackrel{\varepsilon \rightarrow 0}{\longrightarrow} 0$.

(b) If $\lambda_{n}=\tau_{k},\left\|\varphi_{n}^{\varepsilon}-\varepsilon^{-1 / 2} \gamma_{k}\right\|_{H^{1}\left(R_{\varepsilon}\right)} \stackrel{\varepsilon \rightarrow 0}{\longrightarrow} 0$.

The main objective of this work is to study the rates of the convergence of the eigenvalues given by Theorem 2.2.

Our main theorem is the following:

Theorem 2.5. Let $n \in N$ and assume $\lambda_{n}$ is a simple eigenvalue, that is, $\lambda_{n-1}<$ $\lambda_{n}<\lambda_{n+1}$. We have

(a) If $\lambda_{n}=\mu_{k}$, then

$$
\lambda_{n}^{\varepsilon}=\mu_{k}+\varepsilon \cdot \Theta_{\mu_{k}}\left(\phi_{k}(0,0), \phi_{k}(1,0)\right)+o(\varepsilon) .
$$

(b) If $\lambda_{n}=\tau_{k}$, then

$$
\lambda_{n}^{\varepsilon}=\tau_{k}-\frac{\left[g(0) \gamma_{k}^{\prime}(0)\right]^{2}+\left[g(1) \gamma_{k}^{\prime}(1)\right]^{2}}{\pi} \varepsilon|\ln \varepsilon|+o(\varepsilon|\ln \varepsilon|),
$$

where

$$
\Theta_{\lambda}(a, b)=\int_{0}^{1} g\left|\frac{d \xi_{\lambda}^{a, b}}{d x}\right|^{2}-\lambda \int_{0}^{1} g\left|\xi_{\lambda}^{a, b}\right|^{2},
$$

and $\xi_{\lambda}^{a, b}$ is the solution of

$$
\begin{aligned}
-\frac{1}{g}\left(g \xi_{x}\right)_{x} & =\lambda \xi, \quad(0,1), \\
\xi(0) & =a, \quad \xi(1)=b,
\end{aligned}
$$

which exists and is unique for all $\lambda \in \mathbb{R}^{+} \backslash\left\{\tau_{n}\right\}$ and for all $a, b \in \mathbb{R}$.

Notice that the constant $\left(\left[g(0) \gamma_{k}^{\prime}(0)\right]^{2}+\left[g(1) \gamma_{k}^{\prime}(1)\right]^{2}\right) / \pi$ only depends on $g$ and therefore the estimate (2.11) only depends on $R_{\varepsilon}$. Thus, for different $\Omega$ but without changing $R_{\varepsilon}$ we will obtain the same expression. Notice also that the constant $\boldsymbol{\theta}_{\mu_{k}}\left(\phi_{k}(0,0), \phi_{k}(1,0)\right)$ depends on both $\Omega$ and $R_{\varepsilon}$. 
If we consider the case $g \equiv 1$, the constant $\Theta_{\mu_{k}}\left(\phi_{k}(0,0), \phi_{k}(1,0)\right)$ is exactly the same one found in [16].

It is important to mention that in case (b) it is always obtained that $\lambda_{n}^{\varepsilon}<$ $\tau_{k}$ for $\varepsilon$ small. Also, taking into account Theorem 2.1 the same asymptotic behavior holds for the quantities $\lambda_{n}^{\varepsilon}-\tau_{k}^{\varepsilon}$.

We will also be able to obtain precise estimates of the behavior of the eigenfunction $\varphi_{n}^{\varepsilon}$ on $\Omega$ and on $R_{\varepsilon}$. Before stating the results in this direction we need to introduce some notation that will be used throughout the paper.

We will denote by $p_{0}=(0,0), p_{1}=(1,0)$ and $p$ will represent a generic point of $\mathbb{R}^{2} . B(p, \rho)$ will represent the open ball centered at $p$ and with radius $\rho$. Denote by $\left(r_{0}, \theta_{0}\right)$ polar coordinates of $\mathbb{R}^{2}$ centered at $p_{0}$ and by $\left(r_{1}, \theta_{1}\right)$ polar coordinates of $\mathbb{R}^{2}$ centered at $p_{1}$. Consider the following (see Figure 5):

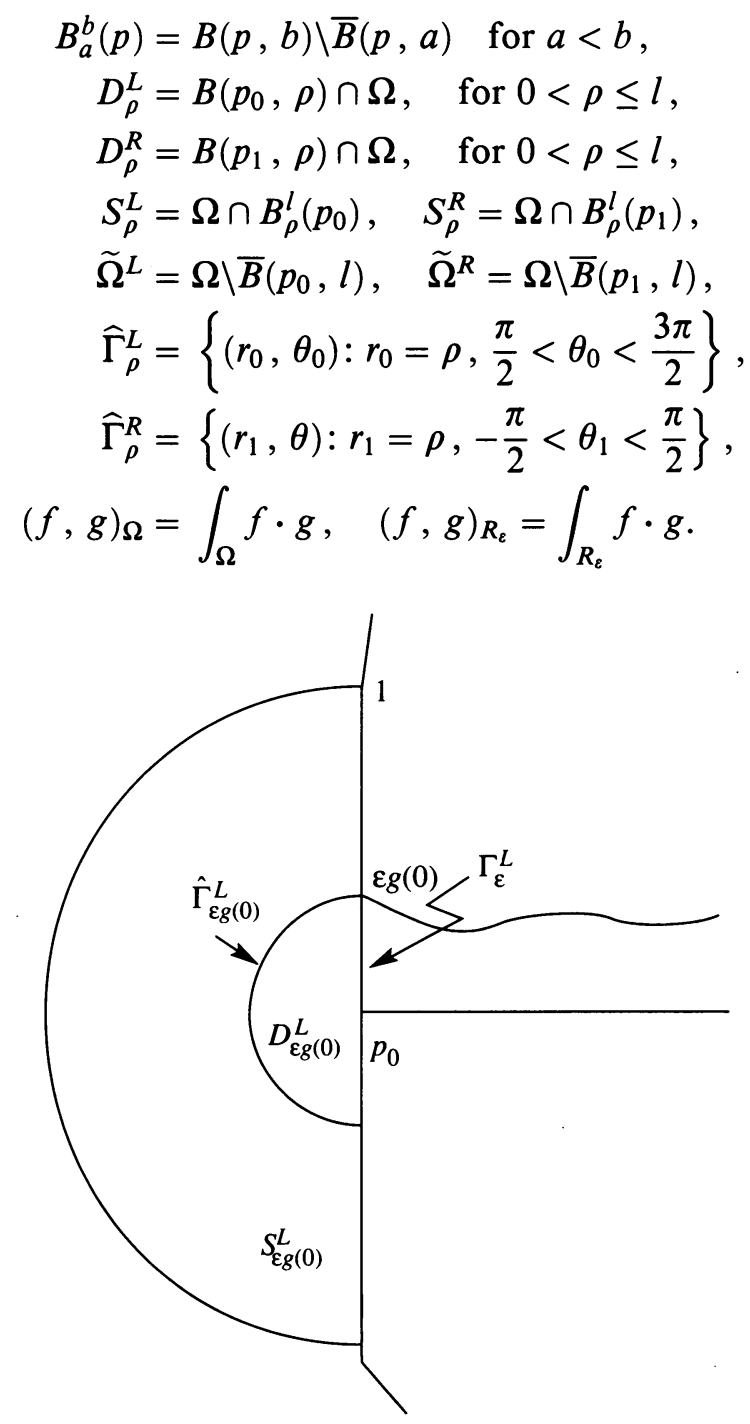

FIGURE 5 
Consider also the following functions:

Definition 2.6. Let $p \in \mathbb{R}$ and let $\zeta_{\varepsilon}^{p}(x, y)$ be the solution of the following problem:

$$
\begin{aligned}
\Delta \zeta & =0, & & B_{\varepsilon}^{l}(p), \\
\zeta & =1, & & \partial B(p, \varepsilon), \\
\zeta & =0, & & \partial B(p, l),
\end{aligned}
$$

where $B(p, a), B(p, b)$ and $B_{a}^{b}(p)$ are defined by (2.14). Consider also

$$
\eta_{\varepsilon}^{L}=\left\{\begin{array}{ll}
0 & \text { in } \widetilde{\Omega}^{L}, \\
\zeta_{\varepsilon g(0)}^{p_{0}} & \text { in } S_{\varepsilon g(0)}^{L}, \\
1 & \text { in } D_{\varepsilon g(0)}^{L},
\end{array} \quad \eta_{\varepsilon}^{R}= \begin{cases}0 & \text { in } \widetilde{\Omega}^{R}, \\
\zeta_{\varepsilon g(1)}^{p_{1}} & \text { in } S_{\varepsilon g(1)}^{R}, \\
1 & \text { in } D_{\varepsilon g(1)}^{R}\end{cases}\right.
$$

Let $\psi_{\varepsilon} \in H^{1}(\Omega)$, and define

$$
\begin{aligned}
& T_{\varepsilon}^{L} \psi_{\varepsilon}=\frac{1}{\varepsilon g(0)} \int_{0}^{\varepsilon g(0)} \psi_{\varepsilon}(0, y) d y, \quad T_{\varepsilon}^{R} \psi_{\varepsilon}=\frac{1}{\varepsilon g(1)} \int_{0}^{\varepsilon g(1)} \psi_{\varepsilon}(1, y) d y, \\
& \widehat{T}_{\varepsilon}^{L} \psi_{\varepsilon}=\frac{1}{\pi} \int_{\pi / 2}^{3 \pi / 2} \psi_{\varepsilon}\left(\varepsilon g(1), \theta_{0}\right) d \theta_{0}, \quad \widehat{T}_{\varepsilon}^{R} \psi_{\varepsilon}=\frac{1}{\pi} \int_{-\pi / 2}^{\pi / 2} \psi_{\varepsilon}\left(\varepsilon g(1), \theta_{1}\right) d \theta_{1} .
\end{aligned}
$$

The estimates for the eigenfunctions are given in the following result.

Theorem 2.7. Let $n \in N$ such that $\lambda_{n-1}<\lambda_{n}<\lambda_{n+1}$ and assume the sign criterion on $\varphi_{n}^{\varepsilon}$ given by Remark 2.4. We have

(a) If $\lambda_{n}=\mu_{k}$, for certain $k \in N$, then

$$
\begin{gathered}
T_{\varepsilon}^{L}\left(\varphi_{n}^{\varepsilon}\right) \stackrel{\varepsilon \rightarrow 0}{\longrightarrow} \phi_{k}\left(p_{0}\right), \quad T_{\varepsilon}^{R}\left(\varphi_{n}^{\varepsilon}\right) \stackrel{\varepsilon \rightarrow 0}{\longrightarrow} \phi_{k}\left(p_{1}\right), \\
\left\|\varphi_{n}^{\varepsilon}-\phi_{k}\right\|_{H^{1}(\Omega)}^{2}=o(\varepsilon), \\
\left\|\varphi_{n}^{\varepsilon}-\xi_{\mu_{k}}^{\phi_{k}}\right\|_{H^{1}\left(R_{\varepsilon}\right)}^{2}=o(\varepsilon),
\end{gathered}
$$

where $\xi_{\mu_{k}}^{\phi_{k}}$ is the solution of $-\frac{1}{g}\left(g \xi^{\prime}\right)^{\prime}=\mu_{k} \xi$ in $(0,1)$ with boundary conditions $\xi(0)=\phi_{k}\left(p_{0}\right), \xi(1)=\phi_{k}\left(p_{1}\right)$.

(b) If $\lambda_{n}=\tau_{k}$, for certain $k \in N$, then

$$
\begin{gathered}
{\left[T_{\varepsilon}^{L}\left(\varphi_{n}^{\varepsilon}\right]^{2}=\left[g(0) \gamma_{k}^{\prime}(0)\right]^{2} \varepsilon|\ln \varepsilon|^{2}+o\left(\varepsilon|\ln \varepsilon|^{2}\right),\right.} \\
{\left[T_{\varepsilon}^{R}\left(\varphi_{n}^{\varepsilon}\right)\right]^{2}=\left[g(1) \gamma_{k}^{\prime}(1)\right]^{2} \varepsilon|\ln \varepsilon|^{2}+o\left(\varepsilon|\ln \varepsilon|^{2}\right),} \\
\left\|\varphi_{n}^{\varepsilon}-\left(T_{\varepsilon}^{L}\left(\varphi_{n}^{\varepsilon}\right) \eta_{\varepsilon}^{L}+T_{\varepsilon}^{R}\left(\varphi_{n}^{\varepsilon}\right) \eta_{\varepsilon}^{R}\right)\right\|_{H^{1}(\Omega)}^{2}=o(\varepsilon|\ln \varepsilon|), \\
\left\|\varphi_{n}^{\varepsilon}-\varepsilon^{-1 / 2} \gamma_{k}\right\|_{H^{1}\left(R_{\varepsilon}\right)}^{2}=o(\varepsilon|\ln \varepsilon|) .
\end{gathered}
$$

A natural tool to obtain the results stated above is the min-max characterization of the eigenvalues, which says that $\lambda_{n}^{\varepsilon}$ can be obtained as

$\lambda_{n}^{\varepsilon}=\min \left\{\frac{\|\nabla \chi\|_{\Omega_{\varepsilon}}^{2}}{\|\chi\|_{\Omega_{\varepsilon}}^{2}} ; \chi \in H^{1}\left(\Omega_{\varepsilon}\right) \chi=0\right.$ in $\left.B,\left(\chi, \varphi_{i}^{\varepsilon}\right)_{\Omega_{\varepsilon}}=0, i=1, \ldots, n-1\right\}$.

It is clear that to obtain upperbounds on the behavior of $\lambda_{n}^{\varepsilon}$ we will need "good" test functions and the closer the test function is to the actual eigenfunction the better estimate is found. Also, once the upperbounds are obtained, 
(Section 4), one needs to deal directly with the eigenfunction, perform a hard analysis of its behavior in $\Omega$ and $R_{\varepsilon}$ and basically end up proving that the test functions originally chosen to get the upperbounds are really close to the actual eigenfunctions. From here we will obtain the lowerbounds and as a byproduct we will also prove Theorem 2.7 (Section 5). This is the basic idea in the proof, and it is clear now that we will need to understand the behavior of the eigenfunctions in $\Omega$ and in $R_{\varepsilon}$.

In order to illustrate how this analysis is done, let us consider the simple case $g \equiv 1$.

If $\varphi_{n}^{\varepsilon}$ is an eigenfunction and we define $\psi_{\varepsilon}(x)=\frac{1}{\varepsilon} \int_{0}^{\varepsilon} \varphi_{n}^{\varepsilon}(x, y) d y$, we will have that $\psi_{\varepsilon}$ satisfies the equation

$$
\begin{aligned}
-\left(\psi_{\varepsilon}\right)_{x x} & =\lambda_{n}^{\varepsilon} \psi_{\varepsilon}, & & x \in(0,1), \\
\psi_{\varepsilon}(0) & =T_{\varepsilon}^{L}\left(\varphi_{n}^{\varepsilon}\right), & & \psi_{\varepsilon}(1)=T_{\varepsilon}^{R}\left(\varphi_{n}^{\varepsilon}\right) ;
\end{aligned}
$$

in other words, $\psi_{\varepsilon}=\xi_{\lambda_{n}^{\varepsilon}}^{a_{\varepsilon}, b_{\varepsilon}}$ where $a_{\varepsilon}=T_{\varepsilon}^{L}\left(\varphi_{n}^{\varepsilon}\right), b_{\varepsilon}=T_{\varepsilon}^{R}\left(\varphi_{n}^{\varepsilon}\right)$ and $\xi_{\lambda}^{a, b}$ is defined by (2.13).

Moreover, from the second Poincaré inequality, we will have that

$$
\left\|\varphi_{n}^{\varepsilon}-\psi_{\varepsilon}\right\|_{R_{\varepsilon}}^{2} \leq C \varepsilon^{2}\left\|\frac{\partial \varphi_{n}^{\varepsilon}}{\partial y}\right\|_{R_{\varepsilon}}^{2}=o\left(\varepsilon^{2}\right) .
$$

Thus, the function $\psi_{\varepsilon}$ is close to the actual eigenfunction $\varphi_{n}^{\varepsilon}$ and this gives us the hint that we should consider the family of functions $\xi_{\lambda}^{a, b}$ as possible test functions on the channel.

For case (a), we know already that $\left.\varphi_{n}^{\varepsilon}\right|_{\Omega} \stackrel{\varepsilon \rightarrow 0}{\longrightarrow} \phi_{k}$. From this, we can "expect" that $T_{\varepsilon}^{L}\left(\varphi_{n}^{\varepsilon}\right) \stackrel{\varepsilon \rightarrow 0}{\longrightarrow} \phi_{k}\left(p_{0}\right)$ and $T_{\varepsilon}^{R}\left(\varphi_{n}^{\varepsilon}\right) \stackrel{\varepsilon \rightarrow 0}{\longrightarrow} \phi_{k}\left(p_{1}\right)$. Moreover, since $\lambda_{n}^{\varepsilon} \stackrel{\varepsilon \rightarrow 0}{\longrightarrow} \mu_{k}$ and $\mu \notin\left\{\tau_{j}\right\}$, we know that $\left\|\xi_{\lambda_{n}^{\varepsilon}}^{a, b}-\xi_{\mu_{k}}^{a, b}\right\|_{H^{1}\left(R_{\varepsilon}\right)}^{2}=o(\varepsilon)$. Therefore, heuristically we can "expect" that $\varphi_{n}^{\varepsilon}$ behave like $\phi_{k}$ in $\Omega$ and like $\xi_{\mu_{k}}^{\phi_{k}\left(p_{0}\right), \phi_{k}\left(p_{1}\right)}$ in $R_{\varepsilon}$.

For case (b), we know that $\left\|\varphi_{n}^{\varepsilon}-\varepsilon^{-1 / 2} \gamma_{k}\right\|_{H^{1}\left(R_{\varepsilon}\right)} \stackrel{\varepsilon \rightarrow 0}{\longrightarrow} 0$ and $\left\|\varphi_{n}^{\varepsilon}\right\|_{H^{1}(\Omega)} \stackrel{\varepsilon \rightarrow 0}{\longrightarrow}$ 0 . If we use as a test function

$$
\Upsilon_{\varepsilon}= \begin{cases}0 & \text { in } \Omega, \\ \varepsilon^{-1 / 2} \gamma_{k} & \text { in } R_{\varepsilon},\end{cases}
$$

we will not go further than proving $\lambda_{n}^{\varepsilon} \leq \tau_{k}$. Moreover, from the fact that an eigenfunction does not vanish identically in an open set one can deduce that a "good" test function for this case must have some nonvanishing and relevant component in $\Omega$. The key point in this case is to analyse the behavior of the eigenfunction in $\Omega$. Since $\lambda_{n}^{\varepsilon} \stackrel{\varepsilon \rightarrow 0}{\longrightarrow} \tau_{k}$ and we are assuming $\tau_{k} \notin\left\{\mu_{n}\right\}$, that is, we do not have a resonance phenomenon in $\Omega$, we will be able to prove that $\left\|\varphi_{n}^{\varepsilon}\right\|_{\Omega}^{2}=o\left(\left\|\nabla \varphi_{n}^{\varepsilon}\right\|_{\Omega}^{2}\right)$ (Corollary 5.2). This result and the fact that $\lambda_{n}^{\varepsilon}$ comes from a minimization problem suggest that $\varphi_{n}^{\varepsilon}$ should behave like a harmonic function with certain values in $\Gamma_{\varepsilon}$ and decaying to zero as we move away from $\Gamma_{\varepsilon}$ (that is, the weight of $\varphi_{n}^{\varepsilon}$ is concentrated around $\Gamma_{\varepsilon}$ ). It must seem clear now that a candidate for the test function must be

$$
\Upsilon_{\varepsilon}= \begin{cases}\alpha_{\varepsilon} \eta_{\varepsilon}^{L}+\beta_{\varepsilon} \eta_{\varepsilon}^{R}, & \text { in } \Omega, \\ h_{\varepsilon}(x)+\varepsilon^{-1 / 2} \gamma_{k}, & \text { in } R_{\varepsilon},\end{cases}
$$


where $\alpha_{\varepsilon}, \beta_{\varepsilon}$ are two real parameters, $h_{\varepsilon}(x)$ is the linear function which satisfies $h_{\varepsilon}(0)=\alpha_{\varepsilon}, h_{\varepsilon}(1)=\beta_{\varepsilon}$, and $\eta_{\varepsilon}^{L}, \eta_{\varepsilon}^{R}$ are defined by (2.16).

\section{Some lemmas}

In this section we want to prove some lemmas that will be used throughout this paper.

Lemma 3.1. The function

$$
\begin{aligned}
\left(\mathbb{R}^{+} \backslash\left\{\tau_{n}\right\}\right) \times \mathbb{R} \times \mathbb{R} \rightarrow C^{1}(0,1), \\
(\lambda, a, b) \rightarrow \xi_{\lambda}^{a, b}
\end{aligned}
$$

is continuous, and for any compact set $K \subset \mathbb{R}^{+} \backslash\left\{\tau_{n}\right\}$ there exists a constant $C(K)$ such that

$$
\left\|\xi_{\lambda}^{a, b}\right\|_{H^{1}(0,1)}^{2} \leq C(K)\left(a^{2}+b^{2}\right), \quad \text { for all }(a, b) \in \mathbb{R}^{2} .
$$

Moreover, if $\lambda_{\varepsilon} \in R \backslash\left\{\tau_{n}\right\}$ and $\lambda_{\varepsilon} \stackrel{\varepsilon \rightarrow 0}{\longrightarrow} \tau_{n}$ for some $\tau_{n}$, we have

$$
\begin{aligned}
\int_{0}^{1} g\left|\xi_{\lambda_{\varepsilon}}^{a, b}\right|^{2} & =\left(\int_{0}^{1} g \xi_{\lambda_{\varepsilon}}^{a, b} \gamma_{n}\right)^{2}+f_{1}(\varepsilon, a, b) \\
& =\frac{\left[-a g(0) \gamma_{n}^{\prime}(0)+b g(1) \gamma_{n}^{\prime}(1)\right]^{2}}{\left(\lambda_{\varepsilon}-\tau_{n}\right)^{2}}+f_{1}(\varepsilon, a, b), \\
\int_{0}^{1} g\left|\frac{d \xi_{\lambda_{\varepsilon}}^{a, b}}{d x}\right|^{2} & =\frac{\lambda_{\varepsilon}^{2}\left[-a g(0) \gamma_{n}^{\prime}(0)+b g(1) \gamma_{n}^{\prime}(1)\right]^{2}}{\tau_{n}\left(\lambda_{\varepsilon}-\tau_{n}\right)^{2}}+f_{2}(\varepsilon, a, b), \\
\Theta_{\lambda_{\varepsilon}}(a, b) & =\frac{\left[-a g(0) \gamma_{n}^{\prime}(0)+b g(1) \gamma_{n}^{\prime}(1)\right]^{2}}{\lambda_{\varepsilon}-\tau_{n}}+f_{3}(\varepsilon, a, b),
\end{aligned}
$$

where $f_{i}(\varepsilon, a, b)$ satisfies the condition that there exists a constant $C$ independent of $\varepsilon, a, b$ such that $\left|f_{i}(\varepsilon, a, b)\right| \leq C\left(a^{2}+b^{2}\right)$, for $i=1,2,3$.

Proof. From the linearity of (2.13), we have that $\xi_{\lambda}^{a, b}=a \xi_{\lambda}^{1,0}+b \xi_{\lambda}^{0,1}$. From this decomposition, the continuity of (3.1) and the bound (3.2) are easily obtained.

Assume now that $\lambda_{\varepsilon} \rightarrow \tau_{n}$, and for simplicity let us denote $\xi=\xi_{\lambda_{\varepsilon}}^{a, b}$.

Via a Fourier decomposition of $\xi$ in terms of the eigenfunctions $\left\{\gamma_{i}\right\}_{i=1}^{\infty}$, that is, $\xi=\sum_{i}\left(g \xi, \gamma_{i}\right)_{L^{2}(0,1)} \gamma_{i}$, we obtain

$$
\int_{0}^{1} g|\xi|^{2}=\sum_{i=1}^{\infty}\left(\int_{0}^{1} g \xi \gamma_{i}\right)^{2}=\left(\int_{0}^{1} g \xi \gamma_{n}\right)^{2}+\sum_{i \neq n}\left(\int_{0}^{1} g \xi \gamma_{i}\right)^{2} .
$$

But since $\xi$ satisfies equation (2.13) and $\gamma_{i}$ satisfies equation (2.3), via elementary integration by parts, we deduce that

$$
\int_{0}^{1} g \xi \gamma_{i}=\frac{\left[g \xi \gamma_{i}^{\prime}\right]_{0}^{1}}{\lambda_{\varepsilon}-\tau_{i}}
$$

where we denote $[f(x)]_{0}^{1}=f(1)-f(0)$. 
Let $h(x)$ be the solution of the following problem:

$$
\begin{aligned}
-\left(g h^{\prime}\right)^{\prime} & =0, & & \text { in }(0,1), \\
h(0) & =a, & & h(1)=b .
\end{aligned}
$$

Notice that $h$ can be found explicitly as

$$
h(x)=a+(b-a) \frac{\int_{0}^{x} \frac{d t}{g(t)}}{\int_{0}^{1} \frac{d t}{g(t)}} .
$$

Since by definition $\xi_{\lambda_{\varepsilon}}=h$ at $x=0,1$, we have $\left[g \xi \gamma_{i}^{\prime}\right]_{0}^{1}=\left[g h \gamma_{i}^{\prime}\right]_{0}^{1}$ and again, integrating by parts, we have $\left[g \xi \gamma_{i}^{\prime}\right]_{0}^{1}=-\tau_{i} \int_{0}^{1} g \gamma_{i} h$.

Hence, if we denote by

$$
f_{1}(\varepsilon, a, b)=\sum_{i \neq n}\left(\int_{0}^{1} g \xi \gamma_{i}\right)^{2}
$$

we have, with the aid of (3.8),

$$
f_{1}(\varepsilon, a, b)=\sum_{i \neq n} \frac{\tau_{i}^{2}}{\left(\lambda_{\varepsilon}-\tau_{i}\right)^{2}}\left(\int_{0}^{1} g \gamma_{i} h\right)^{2} \leq C \int_{0}^{1} g|h|^{2} \leq C\left(a^{2}+b^{2}\right) .
$$

Thus, statements (3.6), (3.7), (3.9) and (3.10) prove (3.3).

To prove (3.4) and (3.5) we proceed in a similar way. Notice that

$$
\Theta_{\lambda_{\varepsilon}}(a, b)=\int_{0}^{1} g\left|\xi^{\prime}\right|^{2}-\lambda_{\varepsilon} \int_{0}^{1} g|\xi|^{2}=\left[g \xi^{\prime} \xi\right]_{0}^{1}
$$

and

$$
\left[g \xi^{\prime} \xi\right]_{0}^{1}=\left[g \xi^{\prime} h\right]_{0}^{1}=-\lambda_{\varepsilon} \int_{0}^{1} g \xi h+\left[g \xi h^{\prime}\right]_{0}^{1}=-\lambda_{\varepsilon} \int_{0}^{1} g \xi h+\tilde{f}(a, b),
$$

where $\tilde{f}(a, b)=\left[g \xi h^{\prime}\right]_{0}^{1}$, which, from (3.8), obviously satisfies $|\tilde{f}(a, b)| \leq$ $C\left(a^{2}+b^{2}\right)$. Moreover, as before, via a Fourier decomposition of $\xi$, we have

$$
\int_{0}^{1} g \xi h=\sum_{i=1}^{\infty} \int_{0}^{1} g \xi \gamma_{i} \int_{0}^{1} g h \gamma_{i}=\sum_{i=1}^{\infty} \frac{\left[g \gamma_{i}^{\prime} \xi\right]_{0}^{1}}{\lambda_{\varepsilon}-\tau_{i}} \int_{0}^{1} g h \gamma_{i} .
$$

Integrating by parts, we have $\left[g \gamma_{i}^{\prime} \xi\right]_{0}^{1}=\left[g \gamma_{i}^{\prime} h\right]_{0}^{1}=-\tau_{i} \int_{0}^{1} g \gamma_{i} h$, and therefore,

$$
\int_{0}^{1} g \xi h=-\frac{\left(\left[g \gamma_{n}^{\prime} \xi\right]_{0}^{1}\right)^{2}}{\left(\lambda_{\varepsilon}-\tau_{n}\right) \tau_{n}}+\sum_{i \neq n} \frac{-\tau_{n}}{\lambda_{\varepsilon}-\tau_{n}}\left(\int_{0}^{1} g \gamma_{i} h\right)^{2}
$$

Hence (3.11), (3.12), (3.13) and (3.14) imply

$$
\begin{aligned}
\boldsymbol{\Theta}_{\lambda_{\varepsilon}}(a, b) & =\lambda_{\varepsilon} \frac{\left(\left[g \gamma_{n}^{\prime} \xi\right]_{0}^{1}\right)^{2}}{\left(\lambda_{\varepsilon}-\tau_{n}\right) \tau_{n}}+\sum_{i \neq n} \frac{\lambda_{\varepsilon} \tau_{n}}{\lambda_{\varepsilon}-\tau_{n}}\left(\int_{0}^{1} g \gamma_{i} h\right)^{2}+\tilde{f}(a, b) \\
& =\frac{\left(\left[g \gamma_{n}^{\prime} \xi\right]_{0}^{1}\right)^{2}}{\lambda_{\varepsilon}-\tau_{n}}+\left(\left[g \gamma_{n}^{\prime} \xi\right]_{0}^{1}\right)^{2}+\sum_{i \neq n} \frac{\lambda_{\varepsilon} \tau_{n}}{\lambda_{\varepsilon}-\tau_{n}}\left(\int_{0}^{1} g \gamma_{i} h\right)^{2}+\tilde{f}(a, b) \\
& =\frac{\left(\left[g \gamma_{n}^{\prime} \xi\right]_{0}^{1}\right)^{2}}{\lambda_{\varepsilon}-\tau_{n}}+f_{3}(\varepsilon, a, b),
\end{aligned}
$$


where

$$
f_{3}(\varepsilon, a, b)=\left(\left[g \gamma_{n}^{\prime} \xi\right]_{0}^{1}\right)^{2}+\sum_{i \neq n} \frac{\lambda_{\varepsilon} \tau_{n}}{\lambda_{\varepsilon}-\tau_{n}}\left(\int_{0}^{1} g \gamma_{i} h\right)^{2}+\tilde{f}(a, b),
$$

which trivially satisfies $\left|f_{3}(\varepsilon, a, b)\right| \leq C\left(a^{2}+b^{2}\right)$. This proves (3.5). Now (3.4) can be obtained from (3.3) and (3.5). This proves the lemma.

In the proof of the results we will need to consider a change of variables which will transform the domain $R_{\varepsilon}$ into $Q_{\varepsilon}=(0,1) \times(0, \varepsilon)$. This change of variables is given by the following transformation:

$$
L_{\varepsilon}: Q_{\varepsilon} \rightarrow R_{\varepsilon}, \quad(\hat{x}, \hat{y}) \rightarrow(x, y)=(\hat{x}, g(\hat{x}) \hat{y})
$$

(see [11], [1]).

We have the following

Lemma 3.2. $L_{\varepsilon}$ induces a 1-1 transformation between $H^{1}\left(R_{\varepsilon}\right)$ and $H^{1}\left(Q_{\varepsilon}\right)$, that is,

$$
L_{\varepsilon}: H^{1}\left(R_{\varepsilon}\right) \rightarrow H^{1}\left(Q_{\varepsilon}\right), \quad \varphi \rightarrow \hat{\varphi} \equiv \varphi \circ L_{\varepsilon}
$$

which satisfies

$$
\begin{aligned}
& \left(\frac{\partial \hat{\varphi}}{\partial \hat{x}}, \frac{\partial \hat{\varphi}}{\partial \hat{y}}\right)=\left(\frac{\partial \varphi}{\partial x}-y \frac{g^{\prime}(x)}{g(x)} \frac{\partial \varphi}{\partial y}, \frac{1}{g(x)} \frac{\partial \varphi}{\partial y}\right), \\
& \left(\frac{\partial \varphi}{\partial x}, \frac{\partial \varphi}{\partial y}\right)=\left(\frac{\partial \hat{\varphi}}{\partial \hat{x}}+\hat{y} \frac{g^{\prime}(\hat{x})}{g(\hat{x})} \frac{\partial \hat{\varphi}}{\partial \hat{y}}, g(\hat{x}) \frac{\partial \hat{\varphi}}{\partial \hat{y}}\right) .
\end{aligned}
$$

Moreover, there exist constants $c_{1}, c_{2}$, such that for all $\varphi \in H^{1}\left(R_{\varepsilon}\right)$ we have

$$
\int_{R_{\varepsilon}} \varphi(x, y) d x d y=\int_{Q_{\varepsilon}} g(\hat{x}) \hat{\varphi}(\hat{x}, \hat{y}) d \hat{x} d \hat{y}
$$

and

Proof. Trivial.

$$
\begin{aligned}
& c_{1}\|\hat{\varphi}\|_{L^{2}\left(Q_{\varepsilon}\right)}^{2} \leq\|\varphi\|_{L^{2}\left(R_{\varepsilon}\right)}^{2} \leq c_{2}\|\hat{\varphi}\|_{L^{2}\left(Q_{\varepsilon}\right)}^{2}, \\
& c_{1}\|\hat{\varphi}\|_{H^{1}\left(Q_{\varepsilon}\right)}^{2} \leq\|\varphi\|_{H^{1}\left(R_{\varepsilon}\right)}^{2} \leq c_{2}\|\hat{\varphi}\|_{H^{1}\left(Q_{\varepsilon}\right)}^{2}
\end{aligned}
$$

An important operator will be the averaging in the $y$ direction of a function defined on $R_{\varepsilon}$, which is defined by

$$
\begin{gathered}
M: H^{1}\left(R_{\varepsilon}\right) \rightarrow H^{1}(0,1), \\
\varphi \rightarrow M \varphi(x)=\frac{1}{\varepsilon g(x)} \int_{0}^{\varepsilon g(x)} \varphi(x, y) d y \equiv \frac{1}{\varepsilon} \int_{0}^{\varepsilon} g(\hat{x}) \hat{\varphi}(\hat{x}, \hat{y}) d \hat{y} .
\end{gathered}
$$

Lemma 3.3. There exists a positive constant $C$ such that, if $\psi_{\varepsilon} \in H^{1}\left(R_{\varepsilon}\right)$, we have

$$
\begin{gathered}
\left\|\psi_{\varepsilon}\right\|_{R_{\varepsilon}}^{2}=\left\|\psi_{\varepsilon}-M \psi_{\varepsilon}\right\|_{R_{\varepsilon}}^{2}+\left\|M \psi_{\varepsilon}\right\|_{R_{\varepsilon}}^{2}, \\
\left\|M \psi_{\varepsilon}-\psi_{\varepsilon}\right\|_{R_{\varepsilon}}^{2} \leq C \varepsilon^{2}\left\|\frac{\partial \psi_{\varepsilon}}{\partial y}\right\|_{R_{\varepsilon}}^{2} .
\end{gathered}
$$

Proof. Equality (3.24) is straightforward. To prove inequality (3.25) use the second Poincaré inequality.

We have the following 
Lemma 3.4. If $\psi_{\varepsilon} \in H^{1}(\Omega)$ with $\left\|\psi_{\varepsilon}\right\|_{H^{1}(\Omega)} \stackrel{\varepsilon \rightarrow 0}{\longrightarrow} 0$, then

$$
\left|T_{\varepsilon}^{L} \psi_{\varepsilon}-\widehat{T}_{\varepsilon}^{L} \psi_{\varepsilon}\right| \stackrel{\varepsilon \rightarrow 0}{\longrightarrow} 0, \quad\left|T_{\varepsilon}^{R} \psi_{\varepsilon}-\widehat{T}_{\varepsilon}^{R} \psi_{\varepsilon}\right| \stackrel{\varepsilon \rightarrow 0}{\longrightarrow} 0 .
$$

Proof. Let us prove the first statement of (3.26). Since $\left\|\psi_{\varepsilon}\right\|_{H^{1}(\Omega)} \stackrel{\varepsilon \rightarrow 0}{\longrightarrow} 0$, we have that $\left\|\psi_{\varepsilon}\right\|_{H^{1}\left(D_{\varepsilon g(0)}^{L}\right)} \stackrel{\varepsilon \rightarrow 0}{\longrightarrow} 0$. Let $D^{L}=\varepsilon^{-1} D_{\varepsilon g(0)}^{L}$, and define $\bar{\psi}_{\varepsilon}(x, y)=$ $\psi_{\varepsilon}(\varepsilon x, \varepsilon y)$ for $(x, y) \in D^{L}$ and also

Therefore,

$$
a_{\varepsilon}=\frac{1}{\left|D_{\varepsilon g(0)}^{L}\right|} \int_{D_{\varepsilon g(0)}^{L}} \psi_{\varepsilon}=\frac{1}{\left|D^{L}\right|} \int_{D^{L}} \bar{\psi}_{\varepsilon} .
$$

$$
\begin{aligned}
\left|a_{\varepsilon}-T_{\varepsilon}^{L} \psi_{\varepsilon}\right| & =\frac{1}{\varepsilon g(0)}\left|\int_{0}^{\varepsilon g(0)}\left(a_{\varepsilon}-\psi_{\varepsilon}(0, y)\right) d y\right|=\left|\frac{1}{g(0)} \int_{0}^{g(0)}\left(a_{\varepsilon}-\bar{\psi}_{\varepsilon}(0, y)\right) d y\right| \\
& \leq \frac{1}{g(0)} \int_{0}^{g(0)}\left|a_{\varepsilon}-\bar{\psi}_{\varepsilon}\right| \leq C\left\|a_{\varepsilon}-\bar{\psi}_{\varepsilon}(0, \cdot)\right\|_{L^{2}(0, g(0))} \\
& \leq C\left\|a_{\varepsilon}-\bar{\psi}_{\varepsilon}\right\|_{H^{1}\left(D^{L}\right)}=C\left\|a_{\varepsilon}-\bar{\psi}_{\varepsilon}\right\|_{L^{2}\left(D^{L}\right)}+C\left\|\nabla \bar{\psi}_{\varepsilon}\right\|_{L^{2}\left(D^{L}\right)}
\end{aligned}
$$

and from the second Poincare inequality, $\left\|a_{\varepsilon}-\bar{\psi}_{\varepsilon}\right\|_{L^{2}\left(D^{L}\right)} \leq C\left\|\nabla \bar{\psi}_{\varepsilon}\right\|_{L^{2}\left(D^{L}\right)}$, which implies that $\left|a_{\varepsilon}-T_{\varepsilon}^{L} \psi_{\varepsilon}\right| \leq C\left\|\nabla \bar{\psi}_{\varepsilon}\right\|_{L^{2}\left(D^{L}\right)}$.

Since $D^{L} \subset \mathbb{R}^{2}$ we know that $\left\|\nabla \bar{\psi}_{\varepsilon}\right\|_{L^{2}\left(D^{L}\right)}=\left\|\nabla \psi_{\varepsilon}\right\|_{L^{2}\left(D_{\varepsilon g(0)}^{L}\right)} \stackrel{\varepsilon \rightarrow 0}{\longrightarrow} 0$ and thus $\left|a_{\varepsilon}-T_{\varepsilon}^{L} \psi_{\varepsilon}\right| \stackrel{\varepsilon \rightarrow 0}{\longrightarrow} 0$.

In the same way we can obtain $\left|a_{\varepsilon}-\widehat{T}_{\varepsilon}^{L} \psi_{\varepsilon}\right| \stackrel{\varepsilon \rightarrow 0}{\longrightarrow} 0$ and therefore $\mid T_{\varepsilon}^{L} \psi_{\varepsilon}-$ $\widehat{T}_{\varepsilon}^{L} \psi_{\varepsilon} \mid \stackrel{\varepsilon \rightarrow 0}{\longrightarrow} 0$, which proves the lemma.

Lemma 3.5. The following is satisfied:

$$
\zeta_{\varepsilon}^{p}(x, y)=\frac{\ln \frac{r}{l}}{\ln \frac{\varepsilon}{l}},
$$

and therefore

$$
\begin{aligned}
& \left\|\nabla \zeta_{\varepsilon}^{p}\right\|_{L^{2}\left(B_{\varepsilon}^{l}(p)\right)}^{2}=\frac{2 \pi}{\left|\ln \frac{\varepsilon}{l}\right|}, \\
& \int_{B_{\varepsilon}^{l}(p)} \zeta_{\varepsilon}^{l}=\frac{\pi^{2} l^{2}}{2\left|\ln \frac{\varepsilon}{l}\right|}(1+o(1)), \\
& \left\|\zeta_{\varepsilon}^{p}\right\|_{L^{2}\left(B_{\varepsilon}^{l}(p)\right)}^{2}=\frac{2 \pi l^{2}}{\left|\ln \frac{\varepsilon}{l}\right|^{2}}(1+o(1)) .
\end{aligned}
$$

Proof. Trivial

Lemma 3.6. Let $\psi_{\varepsilon} \in H^{1}(\Omega)$, with $\psi_{\varepsilon} \stackrel{\varepsilon \rightarrow 0}{\longrightarrow} 0$ in $H^{1}(\Omega)$. Assume there exists a $\delta>0$ such that $\left|T_{\varepsilon}^{L} \psi_{\varepsilon}\right| \geq \delta$ (resp. $\left.\left|T_{\varepsilon}^{R} \psi_{\varepsilon}\right| \geq \delta\right)$. Then,

$$
\begin{aligned}
\left\|\nabla \psi_{\varepsilon}\right\|_{D_{l}^{L}}^{2} \geq\left[T_{\varepsilon}^{L} \psi_{\varepsilon}\right]^{2} & \left(\frac{\pi}{|\ln \varepsilon|}+o\left(\frac{1}{|\ln \varepsilon|}\right)\right) \\
& \left(\operatorname{resp.}\left\|\nabla \psi_{\varepsilon}\right\|_{D_{l}^{R}}^{2} \geq\left[T_{\varepsilon}^{R} \psi_{\varepsilon}\right]^{2}\left(\frac{\pi}{|\ln \varepsilon|}+o\left(\frac{1}{|\ln \varepsilon|}\right)\right)\right) .
\end{aligned}
$$


Also, if $\left|T_{\varepsilon}^{L} \psi_{\varepsilon}\right|+\left|T_{\varepsilon}^{R} \psi_{\varepsilon}\right| \geq \delta$, then

$$
\left\|\nabla \psi_{\varepsilon}\right\|_{\Omega}^{2} \geq\left(\left[T_{\varepsilon}^{L} \psi_{\varepsilon}\right]^{2}+\left[T_{\varepsilon}^{R} \psi_{\varepsilon}\right]^{2}\right)\left(\frac{\pi}{|\ln \varepsilon|}+o\left(\frac{1}{|\ln \varepsilon|}\right)\right) .
$$

Proof. Consider the operators

$$
\begin{array}{ll}
N_{\varepsilon}^{L} \psi_{\varepsilon}\left(r_{0}\right)=\frac{1}{\pi} \int_{\pi / 2}^{3 \pi / 2} \psi_{\varepsilon}\left(r_{0}, \theta_{0}\right) d \theta_{0}, & \varepsilon \leq r_{0} \leq l, \\
N_{\varepsilon}^{R} \psi_{\varepsilon}\left(r_{1}\right)=\frac{1}{\pi} \int_{-\pi / 2}^{\pi / 2} \psi\left(r_{1}, \theta_{1}\right) d \theta_{1}, & \varepsilon \leq r_{0} \leq l .
\end{array}
$$

We have

$$
\begin{aligned}
\left\|\nabla \psi_{\varepsilon}\right\|_{D_{l}^{L}}^{2} & \geq\left\|\nabla \psi_{\varepsilon}\right\|_{S_{\varepsilon g(0)}^{L}}^{2} \\
& \geq \min \left\{\left\|\nabla \chi_{\varepsilon}\right\|_{S_{\varepsilon(0)}^{L}}^{2}: N_{\varepsilon}^{L} \chi_{\varepsilon}(l)=N_{\varepsilon}^{L} \psi_{\varepsilon}(l), N_{\varepsilon}^{L} \chi_{\varepsilon}(\varepsilon g(0))=N_{\varepsilon}^{L} \psi_{\varepsilon}(\varepsilon g(0))\right\} \\
& =\left(N_{\varepsilon}^{L} \psi_{\varepsilon}(\varepsilon g(0))-N_{\varepsilon}^{L} \psi_{\varepsilon}(l)\right)^{2} \min \left\{\left\|\nabla \chi_{\varepsilon}\right\|_{S_{\varepsilon(0)}^{L}}^{2}:\right. \\
& \left.\quad N_{\varepsilon}^{L} \chi_{\varepsilon}(l)=0, N_{\varepsilon}^{L} \chi_{\varepsilon}(\varepsilon g(0))=1\right\} \\
& =\left(N_{\varepsilon}^{L} \psi_{\varepsilon}(\varepsilon g(0))-N_{\varepsilon}^{L} \psi_{\varepsilon}(l)\right)^{2}\left\|\nabla \zeta_{\varepsilon g(0)}^{p_{0}}\right\|_{S_{\varepsilon g(0)}^{L}}^{2} \\
& =\left(N_{\varepsilon}^{L} \psi_{\varepsilon}(\varepsilon g(0))-N_{\varepsilon}^{L} \psi_{\varepsilon}(l)\right)^{2} \frac{\pi}{|\ln \varepsilon|}(1+o(1))
\end{aligned}
$$

where we have used Lemma 3.5. Similarly,

$$
\left\|\nabla \psi_{\varepsilon}\right\|_{D_{l}^{R}}^{2} \geq\left(N_{\varepsilon}^{R} \psi_{\varepsilon}(\varepsilon g(1))-N_{\varepsilon}^{R} \psi_{\varepsilon}(l)\right)^{2} \frac{\pi}{|\ln \varepsilon|}(1+o(1))
$$

and therefore,

$$
\begin{aligned}
& \left\|\nabla \psi_{\varepsilon}\right\|_{\Omega}^{2} \geq\left\|\nabla \psi_{\varepsilon}\right\|_{D_{l}^{L}}^{2}+\left\|\nabla \psi_{\varepsilon}\right\|_{D_{l}^{R}}^{2} \\
& \quad \geq\left[\left(N_{\varepsilon}^{L} \psi_{\varepsilon}(\varepsilon g(0))-N_{\varepsilon}^{L} \psi_{\varepsilon}(l)\right)^{2}+\left(N_{\varepsilon}^{R} \psi_{\varepsilon}(\varepsilon g(1))-N_{\varepsilon}^{R} \psi_{\varepsilon}(l)\right)^{2}\right] \frac{\pi}{|\ln \varepsilon|}(1+o(1))
\end{aligned}
$$

From the fact that $\psi_{\varepsilon} \stackrel{\varepsilon \rightarrow 0}{\longrightarrow} 0$ in $H^{1}(\Omega)$, it is easy to see, via a trace theorem, that $N_{\varepsilon}^{L} \psi_{\varepsilon}(l) \stackrel{\varepsilon \rightarrow 0}{\longrightarrow} 0$, and $N_{\varepsilon}^{R} \psi_{\varepsilon}(l) \stackrel{\varepsilon \rightarrow 0}{\longrightarrow} 0$. Moreover, noticing that $N_{\varepsilon}^{L} \psi_{\varepsilon}(\varepsilon g(0))=\widehat{T}_{\varepsilon}^{L} \psi_{\varepsilon}, N_{\varepsilon}^{R} \psi_{\varepsilon}(\varepsilon g(1))=\widehat{T}_{\varepsilon}^{R} \psi_{\varepsilon}$ and applying Lemma 3.4 to the estimates found above, we obtain

$$
\begin{gathered}
\left\|\nabla \psi_{\varepsilon}\right\|_{D_{l}^{L}}^{2} \geq\left(\left[T_{\varepsilon}^{L} \psi_{\varepsilon}\right]^{2}+o(1)\right)\left(\frac{\pi}{|\ln \varepsilon|}+o\left(\frac{1}{|\ln \varepsilon|}\right)\right), \\
\left\|\nabla \psi_{\varepsilon}\right\|_{D_{l}^{R}}^{2} \geq\left(\left[T_{\varepsilon}^{R} \psi_{\varepsilon}\right]^{2}+o(1)\right)\left(\frac{\pi}{|\ln \varepsilon|}+o\left(\frac{1}{|\ln \varepsilon|}\right)\right), \\
\left\|\nabla \psi_{\varepsilon}\right\|_{\Omega}^{2} \geq\left(\left[T_{\varepsilon}^{L} \psi_{\varepsilon}\right]^{2}+\left[T_{\varepsilon}^{R} \psi_{\varepsilon}\right]^{2}+o(1)\right)\left(\frac{\pi}{|\ln \varepsilon|}+o\left(\frac{1}{|\ln \varepsilon|}\right)\right) .
\end{gathered}
$$

Now it is clear that if $\left|T_{\varepsilon}^{L} \psi_{\varepsilon}\right| \geq \delta$ (resp. $\left|T_{\varepsilon}^{R} \psi_{\varepsilon}\right| \geq \delta$ ) or $\left|T_{\varepsilon}^{L} \psi_{\varepsilon}\right|+\left|T_{\varepsilon}^{R} \psi_{\varepsilon}\right| \geq \delta$ we obtain the desired inequalities. This proves the lemma. 
Lemma 3.7. We have

$$
\begin{aligned}
& m_{\varepsilon}^{L}=\min \left\{\left\|\nabla \psi_{\varepsilon}\right\|_{L^{2}\left(D_{l}^{L}\right)}^{2}: T_{\varepsilon}^{L} \psi_{\varepsilon}=1, \psi_{\varepsilon}=0 \text { in } \widehat{\Gamma}_{l}^{L}\right\}=\frac{\pi}{|\ln \varepsilon|}+o\left(\frac{1}{|\ln \varepsilon|}\right), \\
& m_{\varepsilon}^{R}=\min \left\{\left\|\nabla \psi_{\varepsilon}\right\|_{L^{2}\left(D_{l}^{R}\right)}^{2}: T_{\varepsilon}^{R} \psi_{\varepsilon}=1, \psi_{\varepsilon}=0 \text { in } \widehat{\Gamma}_{l}^{R}\right\}=\frac{\pi}{|\ln \varepsilon|}+o\left(\frac{1}{|\ln \varepsilon|}\right) .
\end{aligned}
$$

Proof. Obviously, $m_{\varepsilon}^{L} \leq\left\|\nabla \eta_{\varepsilon}^{l}\right\|_{D_{l}^{L}}^{2}=\frac{\pi}{|\ln \varepsilon|}$. With Lemma 3.6 we easily prove $m_{\varepsilon}^{L} \geq \frac{\pi}{|\ln \varepsilon|}(1+o(1))$.

We will need extension operators from $H_{B}^{1}(\Omega)$ to $H_{B}^{1}\left(\Omega_{\varepsilon}\right)$, where we denote by $H_{B}^{1}(\Omega)=\left\{u \in H^{1}(\Omega): u=0\right.$ in $\left.B\right\}$, and similarly for $H_{B}^{1}\left(\Omega_{\varepsilon}\right)$. For this, let $\lambda \in R \backslash\left\{\tau_{n}\right\}$ and $\psi \in H_{B}^{1}(\Omega)$ and define

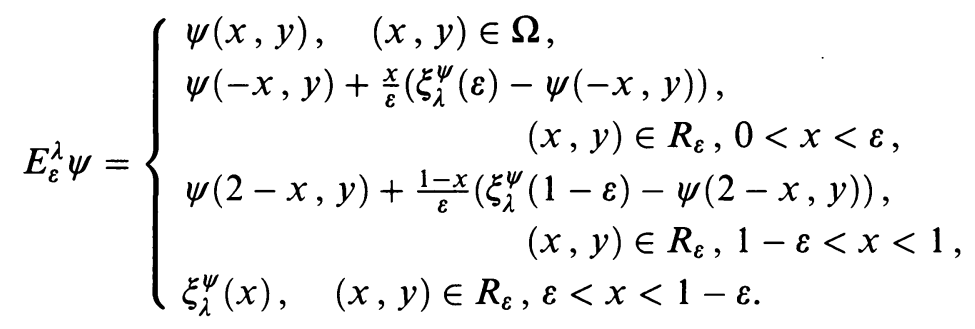

And also

$$
\widetilde{E}_{\varepsilon}^{\lambda} \psi= \begin{cases}\psi(x, y), & (x, y) \in \Omega, \\ \xi_{\lambda}^{\psi}(x), & (x, y) \in R_{\varepsilon},\end{cases}
$$

where we denote by $\xi_{\lambda}^{\psi}$ the solution of (2.13) for $a=\psi\left(p_{0}\right)$ and $b=\psi\left(p_{1}\right)$.

Notice that $E_{\varepsilon}^{\lambda} \psi \in H_{B}^{1}\left(\Omega_{\varepsilon}\right)$, but $\widetilde{E}_{\varepsilon}^{\lambda} \psi \in H_{B}^{1}\left(\Omega \cup R_{\varepsilon}\right)$

Lemma 3.8. If $\varphi \in H_{B}^{1}(\Omega)$, then $E_{\varepsilon}^{\lambda} \varphi \in H_{B}^{1}\left(\Omega_{\varepsilon}\right)$ and $\widetilde{E}_{\varepsilon}^{\lambda} \varphi \in H_{B}^{1}\left(\Omega \cup R_{\varepsilon}\right)$. Moreover

$$
\left\|\nabla E_{\varepsilon}^{\lambda} \varphi-\nabla \widetilde{E}_{\varepsilon}^{\lambda} \varphi\right\|_{\Omega_{\varepsilon}}^{2}=O\left(\varepsilon^{2}\right), \quad\left\|E_{\varepsilon}^{\lambda} \varphi-\widetilde{E}_{\varepsilon}^{\lambda} \varphi\right\|_{\Omega_{\varepsilon}}^{2}=O\left(\varepsilon^{2}\right) .
$$

Proof. Trivial.

Lemma 3.9. Let $i, j \in N$. We have

$$
\begin{aligned}
\left(\nabla \psi_{i}^{\varepsilon}, \nabla \psi_{i}^{\varepsilon}\right)_{\Omega_{\varepsilon}} & =\lambda_{i}+O(\varepsilon), \\
\left(\nabla \psi_{i}^{\varepsilon}, \nabla \psi_{j}^{\varepsilon}\right)_{\Omega_{\varepsilon}} & =O\left(\varepsilon^{1 / 2}\right), \quad i \neq j, \\
\left(\psi_{i}^{\varepsilon}, \psi_{i}^{\varepsilon}\right)_{\Omega_{\varepsilon}} & =1+O(\varepsilon), \\
\left(\psi_{i}^{\varepsilon}, \psi_{j}^{\varepsilon}\right)_{\Omega_{\varepsilon}} & =O\left(\varepsilon^{1 / 2}\right), \quad i \neq j .
\end{aligned}
$$

Proof. Just use the definition of the functions $\psi_{i}^{\varepsilon}$ given by (2.6) and the fact that the measure of the channel $R_{\varepsilon}$ is of the order of $\varepsilon$. Notice that we have to distinguish two cases, according to $\lambda_{i}=\mu_{k}$ and $\lambda_{i}=\tau_{k}$.

\section{UPPER BOUNDS}

In this section we start the proof of Theorem 2.5. We will first obtain upper bounds for $\lambda_{n}^{\varepsilon}$ with the aid of a good test function. Therefore, let us fix $n \in N$ and assume $\lambda_{n-1}<\lambda_{n}<\lambda_{n+1}$. We will need to distinguish two cases: 
(a) Assume $\lambda_{n}=\mu_{k}$ for certain $k \in N$. Consider the following function: $\chi_{\varepsilon}=E_{\varepsilon}^{\mu_{k}} \phi_{k}$ where $E_{\varepsilon}^{\lambda}$ is defined in (3.34), and $\phi_{k}$ is the eigenfunction corresponding to $\mu_{k}$. Notice that $\chi_{\varepsilon}$ cannot be used directly as a test function for $\lambda_{n}^{\varepsilon}$ since it needs to satisfy the orthogonality conditions with respect to the previous $n-1$ eigenfunctions: $\varphi_{1}^{\varepsilon}, \ldots, \varphi_{n-1}^{\varepsilon}$. We do this in the following way. Consider the linear space $\operatorname{span}\left\{\psi_{1}^{\varepsilon}, \ldots, \psi_{n-1}^{\varepsilon}, \chi_{\varepsilon}\right\}$, where $\psi_{i}^{\varepsilon}$ is defined by (2.6), which has dimension $n$ and therefore there exists a nonzero function $\psi_{\varepsilon} \in \operatorname{span}\left\{\psi_{1}^{\varepsilon}, \ldots, \psi_{n-1}^{\varepsilon}, \chi_{\varepsilon}\right\}$ which is orthogonal to the $(n-1)$ dimensional space generated by the first $(n-1)$ eigenfunctions $\varphi_{1}^{\varepsilon}, \ldots, \varphi_{n-1}^{\varepsilon}$. Hence, we can assume the existence of a nonzero vector $a_{1}^{\varepsilon}, \ldots, a_{n}^{\varepsilon}$ such that, if $\psi_{\varepsilon}=\sum_{i=1}^{n-1} a_{i}^{\varepsilon} \psi_{i}^{\varepsilon}+a_{n}^{\varepsilon} \chi_{\varepsilon}$, then $\lambda_{n}^{\varepsilon} \leq\left\|\nabla \psi_{\varepsilon}\right\|_{\Omega_{\varepsilon}}^{2} /\left\|\psi_{\varepsilon}\right\|_{\Omega_{\varepsilon}}^{2}$, or equivalently, $\left\|\nabla \psi_{\varepsilon}\right\|_{\Omega_{\varepsilon}}^{2}-\lambda_{n}^{\varepsilon}\left\|\psi_{\varepsilon}\right\|_{\Omega_{\varepsilon}}^{2} \geq 0$.

But the expression $\left\|\nabla \psi_{\varepsilon}\right\|_{\Omega_{\varepsilon}}^{2}-\lambda_{n}^{\varepsilon}\left\|\psi_{\varepsilon}\right\|_{\Omega_{\varepsilon}}^{2}$ can be regarded as a quadratic form of the column vector $\mathbf{a}=\left(a_{1}^{\varepsilon}, \ldots, a_{n}^{\varepsilon}\right)^{T}$ given by the $n \times n$ symmetric matrix $Q_{\varepsilon}$ with entries

$$
\begin{array}{ll}
q_{i j}=q_{j i}=\left(\nabla \psi_{i}^{\varepsilon}, \nabla \psi_{j}^{\varepsilon}\right)_{\Omega_{\varepsilon}}-\lambda_{n}^{\varepsilon}\left(\psi_{i}^{\varepsilon}, \psi_{j}^{\varepsilon}\right)_{\Omega_{\varepsilon}}, & 1 \leq i, j \leq n-1, \\
q_{i n}=q_{n i}=\left(\nabla \psi_{i}^{\varepsilon}, \nabla \chi_{\varepsilon}\right)_{\Omega_{\varepsilon}}-\lambda_{n}^{\varepsilon}\left(\psi_{i}^{\varepsilon}, \chi_{\varepsilon}\right)_{\Omega_{\varepsilon}}, & 1 \leq i \leq n-1, \\
q_{n n}=\left\|\nabla \chi_{\varepsilon}\right\|_{\Omega_{\varepsilon}}^{2}-\lambda_{n}^{\varepsilon}\left\|\chi_{\varepsilon}\right\|_{\Omega_{\varepsilon}}^{2} .
\end{array}
$$

The existence of a nonzero vector a satisfying $\mathbf{a}^{T} Q_{\varepsilon} \mathbf{a} \geq 0$ is equivalent to the existence of a nonnegative eigenvalue of the matrix $Q_{\varepsilon}$.

It can be seen that the following estimates hold:

$$
\begin{aligned}
& q_{i i}=\lambda_{i}-\lambda_{n}^{\varepsilon}+O(\varepsilon) \leq-\alpha_{i}<0, \quad i=1, \ldots, n-1, \\
& q_{i j}=O\left(\varepsilon^{1 / 2}\right), \quad 1 \leq i, j \leq n-1, i \neq j, \\
& q_{i n}=o\left(\varepsilon^{1 / 2}\right), \quad i=1, \ldots, n-1 .
\end{aligned}
$$

The first two estimates come directly from Lemma 3.9. For the third one we use first the definition of $\chi_{\varepsilon}$ and Lemma 3.8, so that $q_{i n}=\left(\nabla \psi_{i}^{\varepsilon}, \nabla \xi_{\mu_{k}}^{\phi_{k}}\right)_{R_{\varepsilon}}-$ $\lambda_{n}^{\varepsilon}\left(\psi_{\varepsilon}, \xi_{\mu_{k}}^{\phi_{k}}\right)_{R_{\varepsilon}}+O(\varepsilon)$. If $\lambda_{i}=\mu_{s}$ we obtain that $q_{i n}=O(\varepsilon)$, while if $\lambda_{i}=\tau_{s}$ we obtain, via integration by parts, that $\left.q_{i n}=\left(\mu_{k}-\lambda_{n}^{\varepsilon}\right)\left(\gamma_{s}, \xi_{\mu_{k}}^{\phi_{k}}\right)\right)_{R_{\varepsilon}}+O(\varepsilon)=$ $o(1) o\left(\varepsilon^{1 / 2}\right)+O(\varepsilon)=o\left(\varepsilon^{1 / 2}\right)$.

Thus, if there exists a nonnegative eigenvalue of the matrix $Q_{\varepsilon}$, say $e_{\varepsilon}$, we necessarily must have $\operatorname{det}\left(Q_{\varepsilon}-e_{\varepsilon} I\right)=0$. Expanding this determinant by the last column we have

$$
\left(q_{n n}-e_{\varepsilon}\right)\left[\prod_{i=1}^{n-1}\left(q_{i i}-e_{\varepsilon}\right)+O(\varepsilon)\right]+o(\varepsilon)=0,
$$

which implies that $q_{n n}=e_{\varepsilon}+o(\varepsilon)$.

But, from the definition of $\chi_{\varepsilon}$ and Lemma 3.8, we have

$$
q_{n n}=\lambda_{n}-\lambda_{n}^{\varepsilon}+\varepsilon \Theta_{\mu_{k}}\left(\phi_{k}\left(p_{0}\right), \phi_{k}\left(p_{1}\right)\right)+o(\varepsilon),
$$

and therefore, since $e_{\varepsilon} \geq 0$, we must have

$$
\lambda_{n}^{\varepsilon} \leq \mu_{k}+\varepsilon \Theta_{\mu_{k}}\left(\phi_{k}\left(p_{0}\right), \phi_{k}\left(p_{1}\right)\right)+o(\varepsilon),
$$

which gives us the upper bounds for $\lambda_{n}^{\varepsilon}$. 
(b) Now we consider the case where $\lambda_{n}=\tau_{k}$. Let us consider $\alpha_{\varepsilon}$ and $\beta_{\varepsilon}$ two real parameters with $\alpha_{\varepsilon}, \beta_{\varepsilon} \in[-M, M]$, for $M$ sufficiently large but independent of $\varepsilon$. Also, consider the function $h_{\varepsilon}(x)=\alpha_{\varepsilon}(1-x)+\beta_{\varepsilon} x$ defined for $x \in[0,1]$.

We will proceed similarly as we did in case (a). Consider the function

$$
\Upsilon_{\varepsilon}= \begin{cases}\alpha_{\varepsilon} \eta_{l}^{L}+\beta_{\varepsilon} \eta_{l}^{R}, & \text { in } \Omega, \\ h_{\varepsilon}(x)+\varepsilon^{-1 / 2} \gamma_{k}, & \text { in } R_{\varepsilon} .\end{cases}
$$

Again as in case (a) we will take a nonzero function $\psi_{\varepsilon}$ on the $n$-dimensional space $\left[\psi_{1}^{\varepsilon}, \ldots, \psi_{n-1}^{\varepsilon}, \Upsilon_{\varepsilon}\right]$ which is orthogonal to the $(n-1)$-dimensional space $\left[\varphi_{1}^{\varepsilon}, \ldots, \varphi_{n-1}^{\varepsilon}\right]$. This function can be expressed as $\psi_{\varepsilon}=\sum_{i=1}^{n-1} a_{i}^{\varepsilon} \psi_{i}^{\varepsilon}+a_{n}^{\varepsilon} \Upsilon_{\varepsilon}$, for a certain nonzero vector $\mathbf{a}^{T}=\left(a_{1}, \ldots, a_{n}\right)$. From the minimization property of the eigenvalues, we get $\left\|\nabla \psi_{\varepsilon}\right\|_{\Omega_{\varepsilon}}^{2}-\lambda_{n}^{\varepsilon}\left\|\psi_{\varepsilon}\right\|_{\Omega_{\varepsilon}}^{2} \geq 0$.

If we consider the symmetric matrix $Q_{\varepsilon}$ with entries

$$
\begin{aligned}
q_{i j} & =q_{j i}=\left(\nabla \psi_{i}^{\varepsilon}, \nabla \psi_{j}^{\varepsilon}\right)_{\Omega_{\varepsilon}}-\lambda_{n}^{\varepsilon}\left(\psi_{i}^{\varepsilon}, \psi_{j}^{\varepsilon}\right)_{\Omega_{\varepsilon}}, & & 1 \leq i, j \leq n-1, \\
q_{i n} & =q_{n i}=\left(\nabla \psi_{i}^{\varepsilon}, \nabla \Upsilon_{\varepsilon}\right)_{\Omega_{\varepsilon}}-\lambda_{n}^{\varepsilon}\left(\psi_{i}^{\varepsilon}, \Upsilon_{\varepsilon}\right)_{\Omega_{\varepsilon}}, & & i \leq i \leq n-1, \\
q_{n n} & =\left\|\nabla \Upsilon_{\varepsilon}\right\|_{\Omega_{\varepsilon}}^{2}-\lambda_{n}^{\varepsilon}\left\|\Upsilon_{\varepsilon}\right\|_{\Omega_{\varepsilon}}^{2}, & &
\end{aligned}
$$

there must exist a nonnegative eigenvalue of $Q_{\varepsilon}$.

As in the previous case, it can be seen that the following estimates hold:

$$
\begin{aligned}
& q_{i i}=\lambda_{i}-\lambda_{n}^{\varepsilon}+O(\varepsilon) \leq-\alpha_{i}<0, \quad i=1, \ldots, n-1, \\
& q_{i j}=O\left(\varepsilon^{1 / 2}\right), \quad 1 \leq i, j \leq n-1, i \neq j, \\
& \left|q_{i n}\right|=\left(\left|\alpha_{\varepsilon}\right|+\left|\beta_{\varepsilon}\right|\right) O\left(\frac{1}{|\ln \varepsilon|}\right)+O\left(\varepsilon^{1 / 2}\right), \quad i=1, \ldots, n-1 .
\end{aligned}
$$

Therefore, the existence of a nonnegative eigenvalue of the matrix $Q_{\varepsilon}$, say $e_{\varepsilon}$, implies that $\operatorname{det}\left(Q_{\varepsilon}-e_{\varepsilon} I\right)=0$. Expanding this determinant by the last column, we get

$$
\left(q_{n n}-e_{\varepsilon}\right)\left(\prod_{i=1}^{n-1}\left(q_{i i}-e_{\varepsilon}\right)+O(\varepsilon)\right)+O(1)\left[\left(\left|\alpha_{\varepsilon}\right|+\left|\beta_{\varepsilon}\right|\right) O\left(\frac{1}{|\ln \varepsilon|}\right)\right]^{2}=0,
$$

which implies that there exists a positive constant $C$ such that

$$
q_{n n} \geq-C\left(\alpha_{\varepsilon}^{2}+\beta_{\varepsilon}^{2}\right) O\left(\frac{1}{|\ln \varepsilon|^{2}}\right)+O(\varepsilon) .
$$

But evaluating $q_{n n}$, we can obtain

$$
\begin{aligned}
q_{n n}= & \left(\alpha_{\varepsilon}^{2}+\beta_{\varepsilon}^{2}\right) \frac{\pi}{|\ln \varepsilon|}(1+o(1))-2 \alpha_{\varepsilon} g(0) \gamma_{k}^{\prime}(0) \varepsilon^{1 / 2}(1+o(1)) \\
& +2 \beta_{\varepsilon} g(1) \gamma_{k}^{\prime}(1) \varepsilon^{1 / 2}(1+o(1))+\tau_{k}-\lambda_{n}^{\varepsilon}
\end{aligned}
$$

so that (4.3) can be written as

$$
\begin{aligned}
\left(\alpha_{\varepsilon}^{2}+\right. & \left.\beta_{\varepsilon}^{2}\right) \frac{\pi}{|\ln \varepsilon|}(1+o(1))-2 \alpha_{\varepsilon} g(0) \gamma_{k}^{\prime}(0) \varepsilon^{1 / 2}(1+o(1)) \\
& +2 \beta_{\varepsilon} g(1) \gamma_{k}^{\prime}(1) \varepsilon^{1 / 2}(1+o(1))+\tau_{k}-\lambda_{n}^{\varepsilon}+O(\varepsilon) \geq 0 .
\end{aligned}
$$


Since (4.4) is valid for all choices of $\alpha_{\varepsilon}, \beta_{\varepsilon} \in[-M, M]$, we will have that

$$
\begin{aligned}
& \min \left\{\left(\alpha_{\varepsilon}^{2}+\beta_{\varepsilon}^{2}\right) \frac{\pi}{|\ln \varepsilon|}(1+o(1))-2 \alpha_{\varepsilon} g(0) \gamma_{k}^{\prime}(0) \varepsilon^{1 / 2}(1+o(1))\right. \\
& \left.\quad+2 \beta_{\varepsilon} g(1) \gamma_{k}^{\prime}(1) \varepsilon^{1 / 2}(1+o(1)): \alpha_{\varepsilon}, \beta_{\varepsilon} \in[-M, M]\right\} \geq \lambda_{n}^{\varepsilon}-\tau_{k}+O(\varepsilon) .
\end{aligned}
$$

This minimum is attained at the points

$$
\begin{aligned}
& \alpha_{\varepsilon}=\frac{g(0) \gamma_{k}^{\prime}(0)}{\pi} \varepsilon^{1 / 2}|\ln \varepsilon|(1+o(1)) \in[-M, M], \\
& \beta_{\varepsilon}=-\frac{g(1) \gamma_{k}^{\prime}(1)}{\pi} \varepsilon^{1 / 2}|\ln \varepsilon|(1+o(1)) \in[-M, M],
\end{aligned}
$$

and therefore, we obtain

$$
\lambda_{n}^{\varepsilon} \leq \tau_{k}-\frac{\left[g(0) \gamma_{k}^{\prime}(0)\right]^{2}+\left[g(1) \gamma_{k}^{\prime}(1)\right]^{2}}{\pi} \varepsilon|\ln \varepsilon|+o(\varepsilon|\ln \varepsilon|),
$$

which gives us upperbounds on $\lambda_{n}^{\varepsilon}$.

\section{LOWER BOUNDS}

In this section we obtain the lower bounds on the eigenvalues and estimate the eigenfunctions, proving both, Theorem 2.5 and Theorem 2.7. For this we will need to make a careful analysis of the behavior of the eigenfunction on $\Omega$ and on $R_{\varepsilon}$.

The first proposition gives us information on $\Omega$.

Proposition 5.1. Assume that $\mu_{r-1}<\mu_{r}=\cdots=\mu_{s}<\mu_{s+1}$ for certain integers $r \leq s$. Let $n \in N$ such that $\lambda_{n}^{\varepsilon} \stackrel{\varepsilon \rightarrow 0}{\longrightarrow} \mu_{r}$. Then, if we define $\psi_{\varepsilon}=\varphi_{n}^{\varepsilon}-$ $\sum_{i=r}^{s}\left(\varphi_{n}^{\varepsilon}, \phi_{i}\right)_{\Omega} \phi_{i}$, we have

$$
\left\|\psi_{\varepsilon}\right\|_{\Omega}^{2}=o\left(\left\|\nabla \psi_{\varepsilon}\right\|_{\Omega}^{2}\right)
$$

Proof. If $\left\|\psi_{\varepsilon}\right\|_{\Omega}^{2}=0$ there is nothing to prove. Thus, we can assume that $\left\|\psi_{\varepsilon}\right\|_{\Omega}^{2}>0$ for $\varepsilon$ small enough. In this case, (5.1) is equivalent to $\left\|\nabla \psi_{\varepsilon}\right\|_{\Omega}^{2} /\left\|\psi_{\varepsilon}\right\|_{\Omega}^{2}$ $\stackrel{\varepsilon \rightarrow 0}{\longrightarrow}+\infty$. Assume there exist a sequence, that we denote by $\varepsilon$ again, and a constant $C$ such that $\left\|\nabla \psi_{\varepsilon}\right\|_{\Omega}^{2} /\left\|\psi_{\varepsilon}\right\|_{\Omega}^{2} \leq C$. Define $\chi_{\varepsilon}=\psi_{\varepsilon} /\left\|\psi_{\varepsilon}\right\|_{\Omega}$, so that $\chi_{\varepsilon} \in H_{B}^{1}(\Omega),\left\|\chi_{\varepsilon}\right\|_{\Omega}^{2}=1$ and $\left\|\nabla \chi_{\varepsilon}\right\|_{\Omega}^{2} \leq C$.

We can choose a subsequence, denoted again by $\varepsilon$, and a function $\chi \in H_{B}^{1}(\Omega)$ such that $\chi_{\varepsilon} \stackrel{\varepsilon \rightarrow 0}{\longrightarrow} \chi$ weakly in $H^{1}(\Omega)$, strongly in $L^{2}(\Omega)$, so that $\|\chi\|_{\Omega}^{2}=1$ and $\|\nabla \chi\|_{\Omega}^{2} \leq C$. Notice that since $\psi_{\varepsilon}$ is orthogonal to the space generated by $\phi_{r}, \ldots, \phi_{s}$, in $L^{2}(\Omega)$, we will have that $\left(\chi, \phi_{i}\right)_{\Omega}=0$ for $i=r, \ldots, s$.

The function $\chi_{\varepsilon}$ satisfies the following equations:

$$
\begin{aligned}
-\Delta \chi_{\varepsilon} & =\lambda_{n}^{\varepsilon} \chi_{\varepsilon}+\frac{\left(\lambda_{n}^{\varepsilon}-\mu_{r}\right)}{\left\|\psi_{\varepsilon}\right\|_{\Omega}} \sum_{i=r}^{s}\left(\varphi_{n}^{\varepsilon}, \phi_{i}\right)_{\Omega} \phi_{i}, \quad \Omega, \\
\chi_{\varepsilon} & =0, \quad B, \\
\frac{\partial \chi_{\varepsilon}}{\partial n} & =0, \quad \partial \Omega \backslash\left(B \cup \Gamma_{\varepsilon}\right) .
\end{aligned}
$$


Therefore, for any test function $\psi \in C_{0}^{\infty}\left(\bar{\Omega} \backslash\left(B \cup p_{0} \cup p_{1}\right)\right)$ with the property that $\left(\psi, \phi_{i}\right)_{\Omega}=0$ for $i=r, \ldots, s$, we will have $\left(\nabla \chi_{\varepsilon}, \nabla \psi\right)_{\Omega}=\lambda_{n}^{\varepsilon}\left(\chi_{\varepsilon}, \psi\right)_{\Omega}$ for $\varepsilon$ small enough, and going to the limit,

$$
(\nabla \chi, \nabla \psi)_{\Omega}=\mu_{r}(\chi, \psi)_{\Omega} .
$$

Equation (5.3) is valid for all $\psi \in C_{0}^{\infty}\left(\bar{\Omega} \backslash\left(B \cup p_{0} \cup p_{1}\right)\right)$ with the property that $\left(\psi, \phi_{i}\right)_{\Omega}=0$ for $i=r, \ldots, s$. Eventually we want to prove that $(5.3)$ is valid for all $\psi \in H_{B}^{1}(\Omega)$. Consider first the case where $\psi \in H_{B}^{1}(\Omega)$ with the additional condition that $\psi \perp\left[\phi_{r}, \ldots, \phi_{s}\right]$ in $L^{2}(\Omega)$. By density arguments, we can choose a sequence of functions $\psi_{k} \in C_{0}^{\infty}\left(\bar{\Omega} \backslash\left(B \cup p_{0} \cup p_{1}\right)\right)$ such that $\psi_{k} \rightarrow \psi$ in $H^{1}(\Omega)$. Notice that, in this case, $\left(\psi_{k}, \phi_{i}\right)_{\Omega} \rightarrow\left(\psi, \phi_{i}\right)_{\Omega}=0$, for $i=r, \ldots, s$. Choose functions $f_{r}, \ldots, f_{s} \in C_{0}^{\infty}(\Omega)$ with the property that $\left(f_{i}, \phi_{j}\right)_{\Omega}=\delta_{i j}, r \leq i, j \leq s$ which clearly can be found by a dimensionality argument. Consider the function $\bar{\psi}_{k}=\psi_{k}-\sum_{i=r}^{s}\left(\psi_{k}, \phi_{i}\right)_{\Omega} f_{i}$ which belongs to the space $C_{0}^{\infty}\left(\bar{\Omega} \backslash\left(B \cup p_{0} \cup p_{1}\right)\right)$, and obviously satisfies $\left(\bar{\psi}_{k}, \phi_{i}\right)_{\Omega}=0$ for $i=$ $r, \ldots, s$ and $\bar{\psi}_{k} \stackrel{\varepsilon \rightarrow 0}{\longrightarrow} \psi$ in $H_{B}^{1}(\Omega)$. Therefore, $\left(\nabla \chi, \nabla \bar{\psi}_{k}\right)_{\Omega}=\mu_{r}\left(\chi, \bar{\psi}_{k}\right)_{\Omega}$, and going to the limit we conclude that (5.3) is valid for all $\psi \in H_{B}^{1}(\Omega)$ with $\psi \perp\left[\phi_{r}, \ldots, \phi_{s}\right]$.

If $\psi \in H_{B}^{1}(\Omega)$ we define $\tilde{\psi}=\psi-\sum\left(\psi, \phi_{i}\right)_{\Omega} \phi_{i}$ and we will have $(\nabla \chi, \nabla \tilde{\psi})_{\Omega}$ $=\mu_{r}(\chi, \tilde{\psi})_{\Omega}$, but since $\chi$ is orthogonal to $\left[\phi_{r}, \ldots, \phi_{s}\right]$ we get that $(\nabla \chi, \nabla \psi)_{\Omega}$ $=\mu_{r}(\chi, \psi)_{\Omega}$ for $\psi \in H_{B}^{1}(\Omega)$ and this implies that $\chi$ is an eigenfunction corresponding to the eigenvalue $\mu_{r}$ which is orthogonal to the eigenspace generated by $\mu_{r}$. This is a contradiction and the proposition is proved.

Consider the following

Corollary 5.2. Assume $\lambda_{n-1}<\lambda_{n}<\lambda_{n+1}$ and $\lambda_{n}=\tau_{k}$ for certain $k$. Then,

$$
\left\|\varphi_{n}^{\varepsilon}\right\|_{\Omega}^{2}=o\left(\left\|\nabla \varphi_{n}^{\varepsilon}\right\|_{\Omega}^{2}\right)
$$

Proof. Immediate from the previous proposition.

We need to analyse the behavior of the eigenfunctions on the channel $R_{\varepsilon}$. For this, let us begin by defining the function $\xi^{\varepsilon}$ as the solution of the following problem:

$$
\begin{aligned}
-\left(g \xi_{x}\right)_{x} & =\lambda_{n}^{\varepsilon} g \xi, \quad(0,1), \\
\xi(0) & =T_{\varepsilon}^{L}\left(\varphi_{n}^{\varepsilon}\right), \quad \xi(1)=T_{\varepsilon}^{R}\left(\varphi_{n}^{\varepsilon}\right),
\end{aligned}
$$

which is well defined, with the condition that $\lambda_{n}^{\varepsilon} \notin\left\{\tau_{k}\right\}$. We want to end proving that $\varphi_{n}^{\varepsilon}$ and $\xi^{\varepsilon}$ are close in a certain sense in $H^{1}\left(R_{\varepsilon}\right)$. Notice that if $g \equiv 1$ we trivially have that $M \varphi_{n}^{\varepsilon}=\xi^{\varepsilon}$ in $R_{\varepsilon}$.

Let us prove the following

Proposition 5.3. Let $\lambda_{n}^{\varepsilon} \notin\left\{\tau_{k}\right\}$. There exists a constant $C$, independent of $\varepsilon$, such that

$$
\left\|\varphi_{n}^{\varepsilon}-\xi^{\varepsilon}\right\|_{R_{\varepsilon}}^{2} \leq C \varepsilon^{2} \cdot\left\|\frac{\partial \varphi_{n}^{\varepsilon}}{\partial y}\right\|_{R_{\varepsilon}}^{2}\left(1+\sum_{i=1}^{\infty} \frac{\tau_{i}}{\left(\lambda_{n}^{\varepsilon}-\tau_{i}\right)^{2}}\right) .
$$

Proof. First of all, from Lemma 3.3 and the fact that $\xi^{\varepsilon}$ is independent of $y$, we have $\left\|\varphi_{n}^{\varepsilon}-\xi^{\varepsilon}\right\|_{R_{\varepsilon}}^{2}=\left\|\varphi_{n}^{\varepsilon}-M \varphi_{n}^{\varepsilon}\right\|_{R_{\varepsilon}}^{2}+\left\|M \varphi_{n}^{\varepsilon}-\xi^{\varepsilon}\right\|_{R_{\varepsilon}}^{2}$ and also $\left\|\varphi_{n}^{\varepsilon}-M \varphi_{n}^{\varepsilon}\right\|_{R_{\varepsilon}}^{2} \leq$ $C \varepsilon^{2}\left\|\frac{\partial \varphi_{n}^{\varepsilon}}{\partial y}\right\|_{R_{\varepsilon}}^{2}$. 
Via a Fourier decomposition, we have

$$
\left\|M \varphi_{n}^{\varepsilon}-\xi^{\varepsilon}\right\|_{R_{\varepsilon}}^{2}=\varepsilon \int_{0}^{1} g\left|M \varphi_{n}^{\varepsilon}-\xi^{\varepsilon}\right|^{2}=\varepsilon \sum_{i=1}^{\infty}\left[\int_{0}^{1} g\left(M \varphi_{n}^{\varepsilon}-\xi^{\varepsilon}\right) \gamma_{i}\right]^{2},
$$

where $\left\{\gamma_{i}\right\}$ are the eigenfunctions of $-\left(g \gamma^{\prime}\right)^{\prime}=\tau g \gamma$, with $\gamma(0)=\gamma(1)=0$, satisfying $\int_{0}^{1} g \gamma_{i} \gamma_{j}=\delta_{i j}$. Since the function $\xi^{\varepsilon}$ satisfies $-\left(g \xi_{x}^{\varepsilon}\right)_{x}=\lambda_{n}^{\varepsilon} g \xi^{\varepsilon}, \xi^{\varepsilon}(0)=$ $T_{\varepsilon}^{L}\left(\varphi_{n}^{\varepsilon}\right), \xi^{\varepsilon}(1)=T_{\varepsilon}^{R}\left(\varphi_{n}^{\varepsilon}\right)$, we can easily obtain

$$
\int_{0}^{1} g \xi^{\varepsilon} \gamma_{i}=\frac{\left[g \xi^{\varepsilon} \gamma_{i}^{\prime}\right]_{0}^{1}}{\lambda_{n}^{\varepsilon}-\tau_{i}}
$$

Moreover, via elementary integration by parts and using the change of variables given by Lemma 3.2, we have

$$
\tau_{i} \int_{0}^{1} g \gamma_{i} M \varphi_{n}^{\varepsilon}=\lambda_{n}^{\varepsilon} \int_{0}^{1} g \gamma_{i} M \varphi_{n}^{\varepsilon}+\frac{1}{\varepsilon} \int_{0}^{1} \int_{0}^{\varepsilon g(x)} y \frac{g^{\prime}}{g} \frac{d \gamma_{i}}{d x} \frac{\partial \varphi_{n}^{\varepsilon}}{\partial y} d x d y-\left[g \xi^{\varepsilon} \gamma_{i}^{\prime}\right]_{0}^{1} .
$$

Hence

$$
\left[\int_{0}^{1} g\left(M \varphi_{n}^{\varepsilon}-\xi^{\varepsilon}\right) \gamma_{i}\right]^{2}=\frac{1}{\left(\lambda_{n}^{\varepsilon}-\tau_{i}\right)^{2}} \frac{1}{\varepsilon^{2}}\left[\int_{0}^{1} \int_{0}^{\varepsilon g(x)} y \frac{g^{\prime}}{g} \frac{d \gamma_{i}}{d x} \frac{\partial \varphi_{n}^{\varepsilon}}{\partial y} d x d y\right]^{2},
$$

and via Hölder's inequality and taking into account that $\int_{0}^{1} g\left|\gamma_{i}^{\prime}\right|^{2} d x=\tau_{i}$, we have that there will exist a constant $C$ independent of $\varepsilon$ such that

$$
\left[\int_{0}^{1} \int_{0}^{\varepsilon g(x)} y \frac{g^{\prime}}{g} \frac{d \gamma_{i}}{d x} \frac{\partial \varphi_{n}^{\varepsilon}}{\partial y} d x d y\right]^{2} \leq C \varepsilon \tau_{i} \varepsilon^{2}\left\|\frac{\partial \varphi_{n}^{\varepsilon}}{\partial y}\right\|_{R_{\varepsilon}}^{2},
$$

which proves the proposition.

Consider now the following

Proposition 5.4. Let $\lambda_{n}^{\varepsilon} \notin\left\{\tau_{k}\right\}$. There exist $C_{1}, C_{2}>0$ constants independent of $\varepsilon$, such that

$$
\begin{aligned}
\lambda_{n}^{\varepsilon} \geq & \left\|\nabla \varphi_{n}^{\varepsilon}\right\|_{\Omega}^{2}+\lambda_{n}^{\varepsilon}\left\|\varphi_{n}^{\varepsilon}\right\|_{R_{\varepsilon}}^{2}+C_{1}\left\|\frac{\partial \varphi_{n}^{\varepsilon}}{\partial y}\right\|_{R_{\varepsilon}}^{2}+\left\|\frac{\partial \varphi_{n}^{\varepsilon}}{\partial x}-\frac{d \xi^{\varepsilon}}{d x}\right\|_{R_{\varepsilon}}^{2} \\
& -\lambda_{n}^{\varepsilon}\left\|\varphi_{n}^{\varepsilon}-\xi^{\varepsilon}\right\|_{R_{\varepsilon}}^{2}-C_{2} \varepsilon^{3} \int_{0}^{1} g\left|\xi_{x}^{\varepsilon}\right|^{2}+\varepsilon \Theta_{\lambda_{n}^{\varepsilon}}\left(T_{\varepsilon}^{L} \varphi_{n}^{\varepsilon}, T_{\varepsilon}^{R} \varphi_{n}^{\varepsilon}\right) .
\end{aligned}
$$

Proof. Clearly

$$
\lambda_{n}^{\varepsilon}=\left\|\nabla \varphi_{n}^{\varepsilon}\right\|_{\Omega}^{2}+\left\|\frac{\partial \varphi_{n}^{\varepsilon}}{\partial y}\right\|_{R_{\varepsilon}}^{2}+\left\|\frac{\partial \varphi_{n}^{\varepsilon}}{\partial x}\right\|_{R_{\varepsilon}}^{2} .
$$

But

$$
\left\|\frac{\partial \varphi_{n}^{\varepsilon}}{\partial x}\right\|_{R_{\varepsilon}}^{2}=\left\|\frac{\partial \varphi_{n}^{\varepsilon}}{\partial x}-\frac{d \xi^{\varepsilon}}{d x}\right\|_{R_{\varepsilon}}^{2}-\left\|\frac{d \xi^{\varepsilon}}{d x}\right\|_{R_{\varepsilon}}^{2}+2 \int_{R_{\varepsilon}} \frac{\partial \varphi_{n}^{\varepsilon}}{\partial x} \frac{d \xi^{\varepsilon}}{d x} .
$$

Using the change of variables given by Lemma 3.2, we have

$$
\int_{R_{\varepsilon}} \frac{\partial \varphi_{n}^{\varepsilon}}{\partial x} \frac{d \xi^{\varepsilon}}{d x}=\int_{0}^{1} \int_{0}^{\varepsilon} g \frac{\partial\left(\varphi_{n}^{\varepsilon} \circ L_{\varepsilon}\right)}{\partial \hat{x}} \frac{d \xi^{\varepsilon}}{d \hat{x}}-\int_{0}^{1} \int_{0}^{\varepsilon} g^{\prime} \hat{y} \frac{\partial\left(\varphi_{n}^{\varepsilon} \circ L_{\varepsilon}\right)}{\partial \hat{y}} \frac{d \xi^{\varepsilon}}{d \hat{x}}
$$


and via integration by parts,

$$
\int_{0}^{1} \int_{0}^{\varepsilon} g \frac{\partial\left(\varphi_{n}^{\varepsilon} \circ L_{\varepsilon}\right)}{\partial \hat{x}} \frac{d \xi^{\varepsilon}}{d \hat{x}}=\lambda_{n}^{\varepsilon} \int_{R_{\varepsilon}} \varphi_{n}^{\varepsilon} \xi^{\varepsilon}+\varepsilon \Theta_{\lambda_{n}^{\varepsilon}}\left(T_{\varepsilon}^{L} \varphi_{n}^{\varepsilon}, T_{\varepsilon}^{R} \varphi_{n}^{\varepsilon}\right) .
$$

Moreover,

$$
2 \int_{R_{\varepsilon}} \varphi_{n}^{\varepsilon} \xi^{\varepsilon}=-\left\|\varphi_{n}^{\varepsilon}-\xi^{\varepsilon}\right\|_{R_{\varepsilon}}^{2}+\left\|\varphi_{n}^{\varepsilon}\right\|_{R_{\varepsilon}}^{2}+\left\|\xi^{\varepsilon}\right\|_{R_{\varepsilon}}^{2} .
$$

Statements (5.8)-(5.12) give us

$$
\begin{aligned}
\lambda_{n}^{\varepsilon}= & \left\|\nabla \varphi_{n}^{\varepsilon}\right\|_{\Omega}^{2}+\lambda_{n}^{\varepsilon}\left\|\varphi_{n}^{\varepsilon}\right\|_{R_{\varepsilon}}^{2}+\left\|\frac{\partial \varphi_{n}^{\varepsilon}}{\partial y}\right\|_{R_{\varepsilon}}^{2}+\left\|\frac{\partial \varphi_{n}^{\varepsilon}}{\partial x}-\frac{d \xi^{\varepsilon}}{d x}\right\|_{R_{\varepsilon}}^{2}-\lambda_{n}^{\varepsilon}\left\|\varphi_{n}^{\varepsilon}-\xi^{\varepsilon}\right\|_{R_{\varepsilon}}^{2} \\
& -2 \int_{0}^{1} \int_{0}^{\varepsilon} g^{\prime} \hat{y} \frac{\partial\left(\varphi_{n}^{\varepsilon} \circ L_{\varepsilon}\right)}{\partial \hat{y}} \frac{d \xi^{\varepsilon}}{d \hat{x}}+\varepsilon \Theta_{\lambda_{n}^{\varepsilon}}\left(T_{\varepsilon}^{L} \varphi_{n}^{\varepsilon}, T_{\varepsilon}^{R} \varphi_{n}^{\varepsilon}\right) .
\end{aligned}
$$

But from Hölder's inequality,

$$
\left|2 \int_{0}^{1} \int_{0}^{\varepsilon} g^{\prime} \hat{y} \frac{\partial\left(\varphi_{n}^{\varepsilon} \circ L_{\varepsilon}\right)}{\partial \hat{y}} \frac{d \xi^{\varepsilon}}{d \hat{x}}\right| \leq 2 C\left\|\frac{\partial \varphi_{n}^{\varepsilon}}{\partial y}\right\|_{R_{\varepsilon}} \varepsilon^{3 / 2}\left[\int_{0}^{1} g\left|\xi_{x}^{\varepsilon}\right|^{2}\right]^{1 / 2},
$$

and using that for all $\alpha, \beta$ and for all $a \neq 0,2 \alpha \beta \leq a^{2} \alpha^{2}+\beta^{2} / a^{2}$, we get

$$
\left|2 \int_{0}^{1} \int_{0}^{\varepsilon} g^{\prime} \hat{y} \frac{\partial\left(\varphi_{n}^{\varepsilon} \circ L_{\varepsilon}\right)}{\partial \hat{y}} \frac{d \xi^{\varepsilon}}{d \hat{x}}\right| \leq C^{2} a^{2}\left\|\frac{\partial \varphi_{n}^{\varepsilon}}{\partial y}\right\|_{R_{\varepsilon}}^{2}+\frac{1}{a^{2}} \varepsilon^{3} \int_{0}^{1} g\left|\xi_{x}^{\varepsilon}\right|^{2} .
$$

Choosing $a$ such that $1-C^{2} a^{2}=C_{1}>0$ and denoting by $C_{2}=\frac{1}{a^{2}}$ we conclude the proof of the proposition.

Now we are in a position to obtain the lowerbounds of $\lambda_{n}^{\varepsilon}$. For this, we will use in an essential way the previous propositions, Corollary 5.2 and the already obtained upperbounds of $\lambda_{n}^{\varepsilon}$. We will assume that $\lambda_{n-1}<\lambda_{n}<\lambda_{n+1}$ and will need to distinguish two different cases.

(a) Assume $\lambda_{n}=\mu_{k}$ for certain $k$. In this case it is clear that there exists a constant $C$ such that $\sum_{i=1}^{\infty} \frac{\tau_{i}}{\left(\lambda_{n}^{\varepsilon}-\tau_{i}\right)^{2}} \leq C$. Therefore, Proposition 5.3 tells us that

$$
\left\|\varphi_{n}^{\varepsilon}-\xi^{\varepsilon}\right\|_{R_{\varepsilon}}^{2} \leq C \varepsilon^{2}\left\|\frac{\partial \varphi_{n}^{\varepsilon}}{\partial y}\right\|_{R_{\varepsilon}}^{2} .
$$

From (5.14), Proposition 5.4 and the fact that $\left\|\varphi_{n}^{\varepsilon}\right\|_{R_{\varepsilon}}^{2}=1-\left\|\varphi_{n}^{\varepsilon}\right\|_{\Omega}^{2}$, we obtain

$$
\begin{aligned}
0 \geq & \left\|\nabla \varphi_{n}^{\varepsilon}\right\|_{\Omega}^{2}-\lambda_{n}^{\varepsilon}\left\|\varphi_{n}^{\varepsilon}\right\|_{\Omega}^{2}+\widetilde{C}_{1}\left\|\frac{\partial \varphi_{n}^{\varepsilon}}{\partial y}\right\|_{R_{\varepsilon}}^{2}+\left\|\frac{\partial \varphi_{n}^{\varepsilon}}{\partial x}-\frac{d \xi^{\varepsilon}}{d x}\right\|_{R_{\varepsilon}}^{2} \\
& -C_{2} \varepsilon^{3} \int_{0}^{1} g\left|\xi_{x}^{\varepsilon}\right|^{2}+\varepsilon \Theta_{\lambda_{n}^{\varepsilon}}\left(T_{\varepsilon}^{L} \varphi_{n}^{\varepsilon}, T_{\varepsilon}^{R} \varphi_{n}^{\varepsilon}\right) .
\end{aligned}
$$

Denote by $b_{n}^{\varepsilon}=\left(\varphi_{n}^{\varepsilon}, \phi_{k}\right)_{\Omega}$. We know from Remark 2.4 that $b_{n}^{\varepsilon}=1+o(1)$. We have $\left\|\nabla \varphi_{n}^{\varepsilon}\right\|_{\Omega}^{2}=\left\|\nabla \varphi_{n}^{\varepsilon}-b_{n}^{\varepsilon} \nabla \phi_{k}\right\|_{\Omega}^{2}+\left[b_{n}^{\varepsilon}\right]^{2} \mu_{k}$ and $\left\|\varphi_{n}^{\varepsilon}\right\|_{\Omega}^{2}=\left\|\varphi_{n}^{\varepsilon}-b_{n}^{\varepsilon} \nabla \phi_{k}\right\|_{\Omega}^{2}+$ $\left[b_{n}^{\varepsilon}\right]^{2}$. But from Proposition 5.1 we have $\left\|\varphi_{n}^{\varepsilon}-b_{n}^{\varepsilon} \phi_{k}\right\|_{\Omega}^{2}=o\left(\left\|\nabla \varphi_{n}^{\varepsilon}-b_{n}^{\varepsilon} \nabla \phi_{k}\right\|_{\Omega}^{2}\right)$. 
Therefore, we obtain

$$
\begin{aligned}
{\left[b_{n}^{\varepsilon}\right]^{2}\left(\lambda_{n}^{\varepsilon}-\mu_{k}\right) \geq } & \left\|\nabla \varphi_{n}^{\varepsilon}-b_{n}^{\varepsilon} \nabla \phi_{k}\right\|_{\Omega}^{2}(1+o(1))+\varepsilon \Theta_{\lambda_{n}^{\varepsilon}}\left(T_{\varepsilon}^{L}\left(\varphi_{n}^{\varepsilon}\right), T_{\varepsilon}^{R}\left(\varphi_{n}^{\varepsilon}\right)\right) \\
& -C_{2} \varepsilon^{3} \int_{0}^{1} g\left|\xi_{x}^{\varepsilon}\right|^{2}+S(\varepsilon)
\end{aligned}
$$

where we denote $S(\varepsilon)=\widetilde{C}_{1}\left\|\frac{\partial \varphi_{n}^{e}}{\partial y}\right\|_{R_{\varepsilon}}^{2}+\left\|\frac{\partial \varphi_{n}^{\varepsilon}}{\partial x}-\frac{d \xi^{\varepsilon}}{d x}\right\|_{R_{\varepsilon}}^{2} \geq 0$.

Let us prove now that $T_{\varepsilon}^{L}\left(\varphi_{n}^{\varepsilon}\right) \stackrel{\varepsilon \rightarrow 0}{\longrightarrow} \phi_{k}\left(p_{0}\right), T_{\varepsilon}^{R}\left(\varphi_{n}^{\varepsilon}\right) \stackrel{\varepsilon \rightarrow 0}{\longrightarrow} \phi_{k}\left(p_{1}\right)$ or equivalently $T_{\varepsilon}^{L}\left(\varphi_{n}^{\varepsilon}-b_{n}^{\varepsilon} \phi_{k}\right) \stackrel{\varepsilon \rightarrow 0}{\longrightarrow} 0, T_{\varepsilon}^{R}\left(\varphi_{n}^{\varepsilon}-b_{n}^{\varepsilon} \phi_{k}\right) \stackrel{\varepsilon \rightarrow 0}{\longrightarrow} 0$. Assume this is not the case, so that there exists a positive $\delta$ such that

$$
M_{\varepsilon}=\max \left\{\left|T_{\varepsilon}^{L}\left(\varphi_{n}^{\varepsilon}\right)-b_{n}^{\varepsilon} \phi_{k}(0)\right|,\left|T_{\varepsilon}^{R}\left(\varphi_{n}^{\varepsilon}\right)-b_{n}^{\varepsilon} \phi_{k}(1)\right|\right\} \geq \delta
$$

From Lemma 3.6 it is clear that $\left\|\nabla \varphi_{n}^{\varepsilon}-b_{n}^{\varepsilon} \nabla \phi_{k}\right\|_{\Omega}^{2} \geq M_{\varepsilon}^{2} \frac{\pi}{|\ln \varepsilon|}(1+o(1))$ and from (3.2) and taking into account that $\lambda_{n} \notin\left\{\tau_{n}\right\}$ we have that there exists a constant $C$ independent of $\varepsilon$ such that

$$
\left|\Theta_{\lambda_{n}^{\varepsilon}}\left(T_{\varepsilon}^{L}\left(\varphi_{n}^{\varepsilon}\right), T_{\varepsilon}^{R}\left(\varphi_{n}^{\varepsilon}\right)\right)\right| \leq C\left(\left|T_{\varepsilon}^{L}\left(\varphi_{n}^{\varepsilon}\right)\right|^{2}+\left|T_{\varepsilon}^{R}\left(\varphi_{n}^{\varepsilon}\right)\right|^{2}\right) \leq \widetilde{C}\left(M_{\varepsilon}^{2}+1\right),
$$

and similarly

$$
\int_{0}^{1} g\left|\xi_{x}^{\varepsilon}\right|^{2} \leq C\left(M_{\varepsilon}^{2}+1\right)
$$

Therefore (5.16) can be written as

$$
\left[b_{n}^{\varepsilon}\right]^{2}\left(\lambda_{n}^{\varepsilon}-\mu_{k}\right) \geq \delta^{2} \frac{\pi}{|\ln \varepsilon|}(1+o(1))+O(\varepsilon) \geq \frac{c}{|\ln \varepsilon|},
$$

for certain positive constant $c$. Statement (5.17) is in contradiction with the upperbounds of $\lambda_{n}^{\varepsilon}$ given by (4.1).

Therefore we have

$$
T_{\varepsilon}^{L}\left(\varphi_{n}^{\varepsilon}\right) \stackrel{\varepsilon \rightarrow 0}{\longrightarrow} \phi_{k}\left(p_{0}\right), \quad T_{\varepsilon}^{R}\left(\varphi_{n}^{\varepsilon}\right) \stackrel{\varepsilon \rightarrow 0}{\longrightarrow} \phi_{k}\left(p_{1}\right) .
$$

In particular we have proved (2.17) from Theorem 2.7.

From (5.18) and the continuity of $\Theta_{\lambda}(a, b)$, given by Lemma 3.1, we have

$$
\Theta_{\lambda_{n}^{\varepsilon}}\left(T_{\varepsilon}^{L} \varphi_{n}^{\varepsilon}, T_{\varepsilon}^{R} \varphi_{n}^{\varepsilon}\right)=\Theta_{\mu_{k}}\left(\phi_{k}\left(p_{0}\right), \phi_{k}\left(p_{1}\right)\right)+o(1),
$$

and also $\int_{0}^{1} g\left|\xi_{x}^{\varepsilon}\right|^{2} \leq C$, so that (5.16) yields

$$
\begin{aligned}
{\left[b_{n}^{\varepsilon}\right]^{2}\left(\lambda_{n}^{\varepsilon}-\mu_{k}\right) } & \geq\left\|\nabla \varphi_{n}^{\varepsilon}-b_{n}^{\varepsilon} \nabla \phi_{k}\right\|_{\Omega}^{2}+S(\varepsilon)+\varepsilon \Theta_{\mu_{k}}\left(\phi_{k}(0), \phi_{k}(1)\right)+o(\varepsilon) \\
& \geq \varepsilon \Theta_{\mu_{k}}\left(\phi_{k}(0), \phi_{k}(1)\right)+o(\varepsilon)
\end{aligned}
$$

and since $\left[b_{n}^{\varepsilon}\right]^{2}=1+o(1)$, we get

$$
\lambda_{n}^{\varepsilon}-\mu_{k} \geq \varepsilon \Theta_{\mu_{k}}\left(\phi_{k}(0), \phi_{k}(1)\right)+o(\varepsilon),
$$

which gives us the lowerbounds of $\lambda_{n}^{\varepsilon}$. Hence, (5.20) and (4.1) yield

$$
\lambda_{n}^{\varepsilon}-\mu_{k}=\varepsilon \Theta_{\mu_{k}}\left(\phi_{k}(0), \phi_{k}(1)\right)+o(\varepsilon),
$$

which proves $(2.10)$. 
Using (5.21) in (5.19) we get

$$
\begin{gathered}
\left\|\nabla \varphi_{n}^{\varepsilon}-b_{n}^{\varepsilon} \nabla \phi_{k}\right\|_{\Omega}^{2}=o(\varepsilon), \\
\left\|\frac{\partial \varphi_{n}^{\varepsilon}}{\partial y}\right\|_{R_{\varepsilon}}^{2}+\left\|\frac{\partial \varphi_{n}^{\varepsilon}}{\partial x}-\frac{d \xi^{\varepsilon}}{d x}\right\|_{R_{\varepsilon}}^{2}=o(\varepsilon) .
\end{gathered}
$$

But (5.22) and Proposition 4.1 give us

$$
\left\|\varphi_{n}^{\varepsilon}-b_{n}^{\varepsilon} \phi_{k}\right\|_{H^{1}(\Omega)}^{2}=o(\varepsilon)
$$

Moreover (5.23) and (5.14) imply

$$
\left\|\varphi_{n}^{\varepsilon}-\xi^{\varepsilon}\right\|_{H^{1}\left(R_{\varepsilon}\right)}^{2}=o(\varepsilon) \text {. }
$$

The fact that $\lambda_{n}^{\varepsilon} \rightarrow \mu_{k}$, statements (5.18), (5.25) and the continuity of the map (3.1) given by Lemma 3.1 imply $\left\|\varphi_{n}^{\varepsilon}-\xi_{\mu_{k}}^{\phi_{k}}\right\|_{H^{1}\left(R_{\varepsilon}\right)}^{2} \leq 2\left\|\varphi_{n}^{\varepsilon}-\xi^{\varepsilon}\right\|_{H^{1}\left(R_{\ell}\right)}^{2}+$ $2\left\|\xi^{\varepsilon}-\xi_{\mu_{k}}^{\phi_{k}}\right\|_{H^{1}\left(R_{\varepsilon}\right)}^{2}=o(\varepsilon)$, which proves (2.19).

Moreover from (5.24) we get $\left\|\varphi_{n}^{\varepsilon}-\phi_{k}\right\|_{H^{1}(\Omega)}^{2} \leq 2\left\|\varphi_{n}^{\varepsilon}-b_{n}^{\varepsilon} \phi_{k}\right\|_{H^{1}(\Omega)}^{2}+2\left(1-b_{n}^{\varepsilon}\right)^{2}\left\|\phi_{k}\right\|_{H^{1}(\Omega)}^{2} \leq C\left(1-b_{n}^{\varepsilon}\right)^{2}+o(\varepsilon)$.

But, since $b_{n}^{\varepsilon}=1+o(1)$, we have

$$
\begin{aligned}
1-b_{n}^{\varepsilon} & =\frac{1-\left[b_{n}^{\varepsilon}\right]^{2}}{1+b_{n}^{\varepsilon}} \leq C\left(1-\left[b_{n}^{\varepsilon}\right]^{2}\right)=C\left(\left\|\varphi_{n}^{\varepsilon}-b_{n}^{\varepsilon} \phi_{k}\right\|_{\Omega}^{2}+\left\|\varphi_{n}^{\varepsilon}\right\|_{R_{\varepsilon}}^{2}\right) \\
& \leq o(\varepsilon)+C\left(2\left\|\varphi_{n}^{\varepsilon}-\xi_{\mu_{k}}^{\phi_{k}}\right\|_{R_{\varepsilon}}^{2}+2\left\|\xi_{\mu_{k}}^{\phi_{k}}\right\|_{R_{\varepsilon}}^{2}\right)=O(\varepsilon),
\end{aligned}
$$

and therefore $\left\|\varphi_{n}^{\varepsilon}-\phi_{k}\right\|_{H^{1}(\Omega)}^{2}=o(\varepsilon)$, which proves (2.18).

This concludes the proof of case (a) from Theorem 2.5 and Theorem 2.7.

(b) Assume $\lambda_{n}=\tau_{k}$ for certain $k$. For this case we have the upperbounds given by expression (4.5). Notice that although $\lambda_{n}^{\varepsilon} \rightarrow \tau_{k}$ we have from (4.5) that $\lambda_{n}^{\varepsilon} \notin\left\{\tau_{k}\right\}$, since $\left[\gamma_{k}^{\prime}(0) g(0)\right]^{2}+\left[\gamma_{k}^{\prime}(1) g(1)\right]^{2}>0$, because $\gamma_{k}^{\prime}(0) \neq 0$, $\gamma_{k}^{\prime}(1) \neq 0$, from the continuation principle.

To simplify the notation, we will denote $\alpha_{\varepsilon}=T_{\varepsilon}^{L}\left(\varphi_{n}^{\varepsilon}\right)$ and $\beta_{\varepsilon}=T_{\varepsilon}^{R}\left(\varphi_{n}^{\varepsilon}\right)$. Again, from (4.5), we get that

$$
\sum_{i=1}^{\infty} \frac{\tau_{i}}{\left(\lambda_{n}^{\varepsilon}-\tau_{i}\right)^{2}} \leq \frac{\tau_{k}}{\left(\lambda_{n}^{\varepsilon}-\tau_{k}\right)^{2}}+\sum_{i \neq k} \frac{\tau_{i}}{\left(\lambda_{n}^{\varepsilon}-\tau_{i}\right)^{2}} \leq \frac{c_{1}}{\varepsilon^{2}|\ln \varepsilon|^{2}}+c_{2} \leq \frac{c}{\varepsilon^{2}|\ln \varepsilon|^{2}},
$$

and therefore, Proposition 5.3 gives us

$$
\left\|\varphi_{n}^{\varepsilon}-\xi^{\varepsilon}\right\|_{R_{\varepsilon}}^{2} \leq \frac{c}{|\ln \varepsilon|^{2}}\left\|\frac{\partial \varphi_{n}^{\varepsilon}}{\partial y}\right\|_{R_{\varepsilon}}^{2}
$$

Noting that $\frac{c}{|\ln \varepsilon|^{2}} \rightarrow 0$, using (5.26), Corollary 5.2 and Proposition 5.4, we get

$$
\begin{aligned}
0 \geq & \left\|\nabla \varphi_{n}^{\varepsilon}\right\|_{\Omega}^{2}(1+o(1))+\widetilde{C}_{1}\left\|\frac{\partial \varphi_{n}^{\varepsilon}}{\partial y}\right\|_{R_{\varepsilon}}^{2}+\left\|\frac{\partial \varphi_{n}^{\varepsilon}}{\partial x}-\frac{d \xi^{\varepsilon}}{d x}\right\|_{R_{\varepsilon}}^{2} \\
& -C_{2} \varepsilon^{3} \int_{0}^{1} g\left|\xi_{x}^{\varepsilon}\right|^{2}+\varepsilon \Theta_{\lambda_{n}^{\varepsilon}}\left(\alpha_{\varepsilon}, \beta_{\varepsilon}\right) .
\end{aligned}
$$


From (5.27) we can see that we necessarily must have $\alpha_{\varepsilon}^{2}+\beta_{\varepsilon}^{2}>0$, since if $\alpha_{\varepsilon}=\beta_{\varepsilon}=0$ for some $\varepsilon$ small, we will have $\xi^{\varepsilon}=0$ and therefore $\Theta_{\lambda_{n}^{\varepsilon}}\left(\alpha_{\varepsilon}, \beta_{\varepsilon}\right)=$ 0 , so that $(5.27)$ yields

$$
0 \geq\left\|\nabla \varphi_{n}^{\varepsilon}\right\|_{\Omega}^{2}(1+o(1))+\widetilde{C}_{1}\left\|\frac{\partial \varphi_{n}^{\varepsilon}}{\partial y}\right\|_{R_{\varepsilon}}^{2}+\left\|\frac{\partial \varphi_{n}^{\varepsilon}}{\partial x}\right\|_{R_{\varepsilon}}^{2},
$$

which implies that $\varphi_{n}^{\varepsilon}=0$ in $\Omega_{\varepsilon}$, and this is not true.

Moreover, from Lemma 3.1 and statement (4.5), we can see that

$$
\varepsilon^{3} \int_{0}^{1} g\left|\xi_{x}^{\varepsilon}\right|^{2} \leq \frac{c}{|\ln \varepsilon|} \varepsilon\left|\Theta_{\varepsilon}\left(\alpha_{\varepsilon}, \beta_{\varepsilon}\right)\right|+C \varepsilon^{3}\left(\alpha_{\varepsilon}^{2}+\beta_{\varepsilon}^{2}\right) .
$$

Hence, if we denote $S(\varepsilon)=\widetilde{C}_{1}\left\|\frac{\partial \varphi_{n}^{e}}{\partial y}\right\|_{R_{\varepsilon}}^{2}+\left\|\frac{\partial \varphi_{n}^{e}}{\partial x}-\frac{d \xi^{\varepsilon}}{d x}\right\|_{R_{\varepsilon}}^{2}$, inequality (5.27) implies

(5.28) $0 \geq\left\|\nabla \varphi_{n}^{\varepsilon}\right\|_{\Omega}^{2}(1+o(1))+\varepsilon \Theta_{\lambda_{n}^{\varepsilon}}\left(\alpha_{\varepsilon}, \beta_{\varepsilon}\right)(1+o(1))+S(\varepsilon)-C \varepsilon^{3}\left(\alpha_{\varepsilon}^{2}+\beta_{\varepsilon}^{2}\right)$.

Define

$$
\psi_{\varepsilon}=\frac{\varphi_{n}^{\varepsilon}}{\alpha_{\varepsilon}^{2}+\beta_{\varepsilon}^{2}}, \quad \bar{\alpha}_{\varepsilon}=\frac{\alpha_{\varepsilon}}{\sqrt{\alpha_{\varepsilon}^{2}+\beta_{\varepsilon}^{2}}}, \quad \bar{\beta}_{\varepsilon}=\frac{\beta_{\varepsilon}}{\sqrt{\alpha_{\varepsilon}^{2}+\beta_{\varepsilon}^{2}}},
$$

so that $\bar{\alpha}_{\varepsilon}^{2}+\bar{\beta}_{\varepsilon}^{2}=1$. Taking into account Lemma 3.1 and (5.28), we get

$$
\frac{S(\varepsilon)}{\alpha_{\varepsilon}^{2}+\beta_{\varepsilon}^{2}}+\left\|\nabla \psi_{\varepsilon}\right\|_{\Omega}^{2} \leq \frac{\varepsilon+o(\varepsilon)}{\tau_{k}-\lambda_{n}^{\varepsilon}}\left[-\bar{\alpha}_{\varepsilon} g(0) \gamma_{k}^{\prime}(0)+\bar{\beta}_{\varepsilon} g(1) \gamma_{k}^{\prime}(1)\right]^{2}+O(\varepsilon) .
$$

But

$$
\begin{aligned}
& {\left[-\bar{\alpha}_{\varepsilon} g(0) \gamma_{k}^{\prime}(0)+\bar{\beta}_{\varepsilon} g(1) \gamma_{k}^{\prime}(1)\right]^{2}} \\
& \quad \leq \max \left\{\left[-\bar{\alpha} g(0) \gamma_{k}^{\prime}(0)+\bar{\beta} g(1) \gamma_{k}^{\prime}(1)\right]^{2}: \bar{\alpha}^{2}+\bar{\beta}^{2}=1\right\} \\
& \quad=\left[g(0) \gamma_{k}^{\prime}(0)\right]^{2}+\left[g(1) \gamma_{k}^{\prime}(1)\right]^{2},
\end{aligned}
$$

and this maximum is taken only at the point

$$
(\bar{\alpha}, \bar{\beta})= \pm \frac{\left(-g(0) \gamma_{k}^{\prime}(0), g(1) \gamma_{k}^{\prime}(1)\right)}{\sqrt{\left[g(0) \gamma_{k}^{\prime}(0)\right]^{2}+\left[g(1) \gamma_{k}^{\prime}(1)\right]^{2}}} .
$$

Therefore, (5.29) yields

$$
\frac{S(\varepsilon)}{\alpha_{\varepsilon}^{2}+\beta_{\varepsilon}^{2}}+\left\|\nabla \psi_{\varepsilon}\right\|_{\Omega}^{2} \leq \frac{\varepsilon+o(\varepsilon)}{\tau_{k}-\lambda_{n}^{\varepsilon}}\left(\left[g(0) \gamma_{k}^{\prime}(0)\right]^{2}+\left[g(1) \gamma_{k}^{\prime}(1)\right]^{2}\right)+O(\varepsilon) .
$$

From (4.5) and (5.32), we get

$$
\frac{S(\varepsilon)}{\alpha_{\varepsilon}^{2}+\beta_{\varepsilon}^{2}}+\left\|\nabla \psi_{\varepsilon}\right\|_{\Omega}^{2} \leq \frac{\pi}{|\ln \varepsilon|}(1+o(1)) \stackrel{\varepsilon \rightarrow 0}{\longrightarrow} 0
$$

and from Corollary 5.2,

$$
\left\|\psi_{\varepsilon}\right\|_{\Omega}^{2}=\frac{\left\|\varphi_{n}^{\varepsilon}\right\|_{\Omega}^{2}}{\alpha_{\varepsilon}^{2}+\beta_{\varepsilon}^{2}}=\frac{o\left(\left\|\nabla \varphi_{n}^{\varepsilon}\right\|_{\Omega}^{2}\right)}{\alpha_{\varepsilon}^{2}+\beta_{\varepsilon}^{2}}=o\left(\left\|\nabla \psi_{\varepsilon}\right\|_{\Omega}^{2}\right) \stackrel{\varepsilon \rightarrow 0}{\longrightarrow} 0,
$$

so that $\left\|\psi_{\varepsilon}\right\|_{H^{1}(\Omega)} \stackrel{\varepsilon \rightarrow 0}{\longrightarrow} 0$ and we can apply Lemma 3.6 which gives us

$$
\left\|\nabla \psi_{\varepsilon}\right\|_{\Omega}^{2} \geq\left(\left[T_{\varepsilon}^{L} \psi_{\varepsilon}\right]^{2}+\left[T_{\varepsilon}^{R} \psi_{\varepsilon}\right]^{2}\right) \frac{\pi}{|\ln \varepsilon|}(1+o(1))=\frac{\pi}{|\ln \varepsilon|}(1+o(1)) .
$$


Finally (5.33) and (5.34) imply

$$
\begin{gathered}
\left\|\nabla \psi_{\varepsilon}\right\|_{\Omega}^{2}=\frac{\pi}{|\ln \varepsilon|}(1+o(1)), \\
\frac{S(\varepsilon)}{\alpha_{\varepsilon}^{2}+\beta_{\varepsilon}^{2}}=o\left(\frac{1}{|\ln \varepsilon|}\right) .
\end{gathered}
$$

In particular (5.32) and (5.35) imply

$$
\frac{\pi}{|\ln \varepsilon|}(1+o(1)) \leq \frac{\varepsilon+o(\varepsilon)}{\tau_{k}-\lambda_{n}^{\varepsilon}}\left(\left[g(0) \gamma_{k}^{\prime}(0)\right]^{2}+\left[g(1) \gamma_{k}^{\prime}(1)\right]^{2}\right),
$$

or equivalently

$$
\tau_{k}-\lambda_{n}^{\varepsilon} \leq \frac{\left[g(0) \gamma_{k}^{\prime}(0)\right]^{2}+\left[g(1) \gamma_{k}^{\prime}(1)\right]^{2}}{\pi} \varepsilon|\ln \varepsilon|+o(\varepsilon|\ln \varepsilon|),
$$

which, with (4.5), implies

$$
\lambda_{n}^{\varepsilon}=\tau_{k}-\frac{\left[g(0) \gamma_{k}^{\prime}(0)\right]^{2}+\left[g(1) \gamma_{k}^{\prime}(1)\right]^{2}}{\pi} \varepsilon|\ln \varepsilon|+o(\varepsilon|\ln \varepsilon|),
$$

which proves (2.11).

Now we want to obtain the estimates for the eigenfunction. Taking into account (5.36), (5.39), (5.29), and denoting $A=g(0) \gamma_{k}^{\prime}(0), B=g(1) \gamma_{k}^{\prime}(1)$, we get

$$
\frac{\pi}{|\ln \varepsilon|}(1+o(1))=\left\|\nabla \psi_{\varepsilon}\right\|_{\Omega}^{2} \leq \frac{\varepsilon\left[-\bar{\alpha}_{\varepsilon} A+\bar{\beta}_{\varepsilon} B\right]^{2}}{\frac{A^{2}+B^{2}}{\pi} \varepsilon|\ln \varepsilon|(1+o(1))},
$$

which implies

$$
\left[-\bar{\alpha}_{\varepsilon} A+\bar{\beta}_{\varepsilon} B\right]^{2} \geq\left(A^{2}+B^{2}\right)(1+o(1)),
$$

and, therefore $\bar{\alpha}_{\varepsilon}$ and $\bar{\beta}_{\varepsilon}$ must be near the points defined by (5.31). In other words, we must have

$$
\begin{gathered}
\bar{\alpha}_{\varepsilon}=\frac{\mp A}{\sqrt{A^{2}+B^{2}}}(1+o(1)), \quad \bar{\beta}_{\varepsilon}=\frac{ \pm B}{\sqrt{A^{2}+B^{2}}}(1+o(1)), \\
{\left[-\bar{\alpha}_{\varepsilon} A+\bar{\beta}_{\varepsilon} B\right]^{2}=\left(A^{2}+B^{2}\right)(1+o(1)),}
\end{gathered}
$$

and from (5.43) and the definition of $\bar{\alpha}_{\varepsilon}, \bar{\beta}_{\varepsilon}$ we get

$$
\left[-\alpha_{\varepsilon} A+\beta_{\varepsilon} B\right]^{2}=\left(A^{2}+B^{2}\right)\left(\alpha_{\varepsilon}^{2}+\beta_{\varepsilon}^{2}\right)(1+o(1)) .
$$

Moreover, from (5.26) we have $\left\|\varphi_{n}^{\varepsilon}-\xi^{\varepsilon}\right\|_{R_{\varepsilon}}^{2} \stackrel{\varepsilon \rightarrow 0}{\longrightarrow} 0$ and since $\left\|\varphi_{n}^{\varepsilon}\right\|_{R_{\varepsilon}}^{2}=$ $1+o(1)$, we get $\left\|\xi^{\varepsilon}\right\|_{R_{\varepsilon}}^{2}=1+o(1)$. But from Lemma 3.1

$$
\left\|\xi^{\varepsilon}\right\|_{R_{\varepsilon}}^{2}=\varepsilon \int_{0}^{1} g\left|\xi^{\varepsilon}\right|^{2}=\varepsilon\left[\frac{\left[-\alpha_{\varepsilon} A+\beta_{\varepsilon} B\right]^{2}}{\left(\lambda_{n}^{\varepsilon}-\tau_{k}\right)^{2}}+f_{1}\left(\varepsilon, \alpha_{\varepsilon}, \beta_{\varepsilon}\right)\right],
$$

which implies

$$
1+o(1)=\varepsilon \frac{\left(A^{2}+B^{2}\right)\left(\alpha_{\varepsilon}^{2}+\beta_{\varepsilon}^{2}\right)(1+o(1))}{\frac{\left(A^{2}+B^{2}\right)^{2}}{\pi^{2}} \varepsilon^{2}|\ln \varepsilon|^{2}(1+o(1))}+\varepsilon f_{1}\left(\varepsilon, \alpha_{\varepsilon}, \beta_{\varepsilon}\right),
$$


or equivalently,

$$
\alpha_{\varepsilon}^{2}+\beta_{\varepsilon}^{2}=\frac{A^{2}+B^{2}}{\pi^{2}} \varepsilon|\ln \varepsilon|^{2}+o\left(\varepsilon|\ln \varepsilon|^{2}\right)
$$

Hence,

$$
\begin{aligned}
& {\left[T_{\varepsilon}^{L}\left(\varphi_{n}^{\varepsilon}\right)\right]^{2}=\frac{A^{2}}{\pi^{2}} \varepsilon|\ln \varepsilon|^{2}+o\left(\varepsilon|\ln \varepsilon|^{2}\right),} \\
& {\left[T_{\varepsilon}^{R}\left(\varphi_{n}^{\varepsilon}\right)\right]^{2}=\frac{B^{2}}{\pi^{2}} \varepsilon|\ln \varepsilon|^{2}+o\left(\varepsilon|\ln \varepsilon|^{2}\right),}
\end{aligned}
$$

which proves $(2.20)$.

Remark 5.5. Notice that in particular we have proved that

$$
\lambda_{n}^{\varepsilon}-\tau_{k}=\varepsilon \Theta_{\lambda_{n}^{\varepsilon}}\left(T_{\varepsilon}^{L}\left(\varphi_{n}^{\varepsilon}\right), T_{\varepsilon}^{R}\left(\varphi_{n}^{\varepsilon}\right)\right)+o(\varepsilon|\ln \varepsilon|),
$$

since, if we evaluate $\varepsilon \Theta_{\lambda_{n}^{\varepsilon}}\left(T_{\varepsilon}^{L}\left(\varphi_{n}^{\varepsilon}\right), T_{\varepsilon}^{R}\left(\varphi_{n}^{n}\right)\right)$, taking into account Lemma 3.1 and statements (5.44), (5.47) and (5.48), we get

$$
\varepsilon \Theta_{\lambda_{n}^{\varepsilon}}\left(T_{\varepsilon}^{L}\left(\varphi_{n}^{\varepsilon}\right), T_{\varepsilon}^{R}\left(\varphi_{n}^{\varepsilon}\right)\right)=-\frac{A^{2}+B^{2}}{\pi} \varepsilon|\ln \varepsilon|(1+o(1))
$$

which proves (5.49).

Moreover, (5.36) and (5.47) imply

$$
S(\varepsilon)=\left(\alpha_{\varepsilon}^{2}+\beta_{\varepsilon}^{2}\right) o\left(\frac{1}{|\ln \varepsilon|}\right),
$$

and therefore,

$$
\left\|\frac{\partial \varphi_{n}^{\varepsilon}}{\partial y}\right\|_{R_{\varepsilon}}^{2}+\left\|\frac{\partial \varphi_{n}^{\varepsilon}}{\partial x}-\frac{d \xi^{\varepsilon}}{d x}\right\|_{R_{\varepsilon}}^{2}=o(\varepsilon|\ln \varepsilon|)
$$

and with (5.26) we get

$$
\left\|\varphi_{n}^{\varepsilon}-\xi^{\varepsilon}\right\|_{H^{1}\left(R_{\varepsilon}\right)}^{2}=o(\varepsilon|\ln \varepsilon|) .
$$

Moreover, if we define $\chi_{\varepsilon}=\bar{\alpha}_{\varepsilon} \eta_{\varepsilon}^{L}+\bar{\beta}_{\varepsilon} \eta_{\varepsilon}^{R}$, we have

$$
\begin{gathered}
\left\|\nabla \psi_{\varepsilon}-\nabla \chi_{\varepsilon}\right\|_{\Omega}^{2}=2\left\|\nabla \psi_{\varepsilon}\right\|_{\Omega}^{2}+2\left\|\nabla \chi_{\varepsilon}\right\|_{\Omega}^{2}-\left\|\nabla \psi_{\varepsilon}+\nabla \chi_{\varepsilon}\right\|_{\Omega}^{2} \\
=\frac{4 \pi}{|\ln \varepsilon|}(1+o(1))-\left\|\nabla \psi_{\varepsilon}+\nabla \chi_{\varepsilon}\right\|_{\Omega}^{2} .
\end{gathered}
$$

But since $\left\|\psi_{\varepsilon}+\chi_{\varepsilon}\right\|_{H^{1}\left(R_{\varepsilon}\right)} \stackrel{\varepsilon \rightarrow 0}{\longrightarrow} 0$ and $\left[T_{\varepsilon}^{L}\left(\psi_{\varepsilon}+\chi_{\varepsilon}\right)\right]^{2}+\left[T_{\varepsilon}^{R}\left(\psi_{\varepsilon}+\chi_{\varepsilon}\right)\right]^{2}=4$, we have from Lemma 3.6

$$
\left\|\nabla \psi_{\varepsilon}+\nabla \chi_{\varepsilon}\right\|_{\Omega}^{2} \geq \frac{4 \pi}{|\ln \varepsilon|}(1+o(1)),
$$

so that

$$
\left\|\nabla \psi_{\varepsilon}-\nabla \chi_{\varepsilon}\right\|_{\Omega}^{2}=o\left(\frac{1}{|\ln \varepsilon|}\right)
$$


and from (5.55) and (5.47) we have

$$
\left\|\nabla \varphi_{n}^{\varepsilon}-\left(\alpha_{\varepsilon} \nabla \eta_{\varepsilon}^{L}+\beta_{\varepsilon} \nabla \eta_{\varepsilon}^{R}\right)\right\|_{R_{\varepsilon}}^{2}=o(\varepsilon|\ln \varepsilon|)
$$

But trivially we have

$$
\begin{gathered}
\left\|\varphi_{n}^{\varepsilon}-\left(\alpha_{\varepsilon} \eta_{\varepsilon}^{L}+\beta_{\varepsilon} \eta_{\varepsilon}^{R}\right)\right\|_{\Omega}^{2} \leq 2\left\|\varphi_{n}^{\varepsilon}\right\|_{\Omega}^{2}+2\left(\alpha_{\varepsilon}^{2}+\beta_{\varepsilon}^{2}\right)\left\|\eta_{\varepsilon}^{L}\right\|_{\Omega}^{2} \\
=o\left(\left\|\nabla \varphi_{n}^{\varepsilon}\right\|_{\Omega}^{2}\right)+O\left(\varepsilon|\ln \varepsilon|^{2}\right) \frac{1}{|\ln \varepsilon|^{2}}=o(\varepsilon|\ln \varepsilon|)
\end{gathered}
$$

Statements (5.56) and (5.57) prove (2.21).

Finally, let us prove now (2.22). For this let us define first

$$
a_{\varepsilon}=\left(\varphi_{n}^{\varepsilon}, \varepsilon^{-1 / 2} \gamma_{k}\right)_{R_{\varepsilon}}
$$

and evaluate,

$$
\begin{aligned}
\| \varphi_{n}^{\varepsilon}- & a_{\varepsilon} \varepsilon^{-1 / 2} \gamma_{k}\left\|_{R_{\varepsilon}}^{2} \leq\right\| \varphi_{n}^{\varepsilon}-\xi^{\varepsilon}\left\|_{R_{\varepsilon}}^{2}+2\right\| \xi^{\varepsilon}-a_{\varepsilon} \varepsilon^{-1 / 2} \gamma_{k} \|_{R_{\varepsilon}}^{2} \\
\leq & o(\varepsilon|\ln \varepsilon|)+4\left\|\xi^{\varepsilon}-\left(\xi^{\varepsilon}, \varepsilon^{-1 / 2} \gamma_{k}\right)_{R_{\varepsilon}} \varepsilon^{-1 / 2} \gamma_{k}\right\|_{R_{\varepsilon}}^{2} \\
& +\left\|\left(\varphi_{n}^{\varepsilon}-\xi^{\varepsilon}, \varepsilon^{-1 / 2} \gamma_{k}\right)_{R_{\varepsilon}} \varepsilon^{-1 / 2} \gamma_{k}\right\|_{R_{\varepsilon}}^{2} \\
\leq & o(\varepsilon|\ln \varepsilon|)+4\left(\left\|\xi^{\varepsilon}\right\|_{R_{\varepsilon}}^{2}-\left(\xi^{\varepsilon}, \varepsilon^{-1 / 2} \gamma_{k}\right)_{R_{\varepsilon}}^{2}\right)+4\left\|\varphi_{n}^{\varepsilon}-\xi^{\varepsilon}\right\|_{R_{\varepsilon}}^{2} \\
\leq & o(\varepsilon|\ln \varepsilon|)+4 \varepsilon\left[\int_{0}^{1} g\left|\xi^{\varepsilon}\right|^{2}-\left(\int_{0}^{1} g \xi^{\varepsilon} \gamma_{k}\right)^{2}\right]=o(\varepsilon|\ln \varepsilon|),
\end{aligned}
$$

where we have used (5.52) and Lemma 3.1 to estimate $\int_{0}^{1} g\left|\xi^{\varepsilon}\right|^{2}-\left(\int_{0}^{1} g \xi^{\varepsilon} \gamma_{k}\right)^{2}$.

Therefore,

$$
\begin{aligned}
\left\|\varphi_{n}^{\varepsilon}-\varepsilon^{-1 / 2} \gamma_{k}\right\|_{R_{\varepsilon}}^{2} & \leq 2\left\|\varphi_{n}^{\varepsilon}-a_{\varepsilon} \varepsilon^{-1 / 2} \gamma_{k}\right\|_{R_{\varepsilon}}^{2}+2\left(1-a_{\varepsilon}\right)^{2} \\
& \leq o(\varepsilon|\ln \varepsilon|)+2\left(1-a_{\varepsilon}\right)^{2}
\end{aligned}
$$

But, from the sign criterion from Remark 2.4 we know that $a_{\varepsilon}=1+o(1)$. Therefore

$$
\begin{aligned}
0 & <1-a_{\varepsilon}=\frac{1-a_{\varepsilon}^{2}}{1+a_{\varepsilon}} \leq C\left(1-a_{\varepsilon}^{2}\right) \\
& =C\left(\left\|\varphi_{n}^{\varepsilon}-a_{\varepsilon} \varepsilon^{-1 / 2} \gamma_{k}\right\|_{R_{\varepsilon}}^{2}+\left\|\varphi_{n}^{\varepsilon}\right\|_{\Omega}^{2}\right)=o(\varepsilon|\ln \varepsilon|) .
\end{aligned}
$$

Hence (5.59) and (5.60) imply

$$
\left\|\varphi_{n}^{\varepsilon}-\varepsilon^{-1 / 2} \gamma_{k}\right\|_{R_{\varepsilon}}^{2}=o(\varepsilon|\ln \varepsilon|)
$$


Moreover,

$$
\begin{aligned}
\| \nabla \varphi_{n}^{\varepsilon}- & \varepsilon^{-1 / 2} \nabla \gamma_{k}\left\|_{R_{\varepsilon}}^{2}=\right\| \frac{\partial \varphi_{n}^{\varepsilon}}{\partial y}\left\|_{R_{\varepsilon}}^{2}+\right\| \frac{\partial \varphi_{n}^{\varepsilon}}{\partial x}-\varepsilon^{-1 / 2} \frac{d \gamma_{k}}{d x} \|_{R_{\varepsilon}}^{2} \\
= & o(\varepsilon|\ln \varepsilon|)+2\left\|\frac{\partial \varphi_{n}^{\varepsilon}}{\partial x}-a_{\varepsilon} \varepsilon^{-1 / 2} \frac{d \gamma_{k}}{d x}\right\|_{R_{\varepsilon}}^{2}+2\left(1-a_{\varepsilon}\right)^{2}\left\|\varepsilon^{-1 / 2} \frac{d \gamma_{k}}{d x}\right\|_{R_{\varepsilon}}^{2} \\
= & o(\varepsilon|\ln \varepsilon|)+2\left\|\frac{\partial \varphi_{n}^{\varepsilon}}{\partial x}-a_{\varepsilon} \varepsilon^{-1 / 2} \frac{d \gamma_{k}}{d x}\right\|_{R_{\varepsilon}}^{2} \\
\leq & o(\varepsilon|\ln \varepsilon|)+4\left\|\frac{\partial \varphi_{n}^{\varepsilon}}{\partial x}-\frac{d \xi^{\varepsilon}}{d x}\right\|_{R_{\varepsilon}}^{2}+4\left\|\frac{d \xi^{\varepsilon}}{d x}-\left(\varepsilon^{-1 / 2} \gamma_{k}, \xi^{\varepsilon}\right)_{R_{\varepsilon}} \varepsilon^{-1 / 2} \frac{d \gamma_{k}}{d x}\right\|_{R_{\varepsilon}}^{2} \\
& +4\left\|\left(\varphi_{n}^{\varepsilon}-\xi^{\varepsilon}, \varepsilon^{-1 / 2} \gamma_{k}\right)_{R_{\varepsilon}} \varepsilon^{-1 / 2} \frac{d \gamma_{k}}{d x}\right\|_{R_{\varepsilon}}^{2} \\
\leq & o(\varepsilon|\ln \varepsilon|)+4\left\|\frac{d \xi^{\varepsilon}}{d x}-\left(\varepsilon^{-1 / 2} \gamma_{k}, \xi^{\varepsilon}\right)_{R_{\varepsilon} \varepsilon^{-1 / 2}} \frac{d \gamma_{k}}{d x}\right\|_{R_{\varepsilon}}^{2} \\
= & o(\varepsilon|\ln \varepsilon|)+4\left[\varepsilon \Theta_{\lambda_{n}^{\varepsilon}}\left(\alpha_{\varepsilon}, \beta_{\varepsilon}\right)+\left(\tau_{k}-\lambda_{n}^{\varepsilon}\right)\right] \\
& +4 \varepsilon \lambda_{n}^{\varepsilon}\left[\int_{0}^{1} g\left|\xi^{\varepsilon}\right|^{2}-\left(\int_{0}^{1} g \xi^{\varepsilon} \gamma_{k}\right)^{2}\right] \\
= & o(\varepsilon|\ln \varepsilon|),
\end{aligned}
$$

where we have used Remark 5.5 and Lemma 3.1 in the last equality. Obviously, (5.61) and (5.62) prove (2.22). This concludes the proof of Theorem 2.7.

\section{ACKNOWLEDGMENTS}

Part of the initial ideas of this work were developed during the author's stay in the Departamento de Matemáticas y Mecánica at I.I.M.A.S.-U.N.A.M., México D.F. From there, I would like to especially thank Arturo Olvera, Anton Minzoni and Gilberto Flores for their comments and support. I also would like to thank Jack K. Hale for his always good suggestions in these types of problems.

\section{REFERENCES}

1. J. M. Arrieta, Spectral properties of Schrödinger operators under perturbations of the domain, Doctoral Dissertation, Georgia Institute of Technology, 1991.

2. __ Neumann eigenvalue problems on exterior perturbations of the domain, J. Differential Equations 117 (1995).

3. J. M. Arrieta, J. K. Hale and Q. Han, Eigenvalue problems for nonsmoothly perturbed domains, J. Differential Equations 91 (1991), 24-52.

4. I. Babuska and R. Vyborny, Continuous dependence of eigenvalues on the domains, Czechoslovak Math. J. 15 (1965), 169-178.

5. J. T. Beale, Scattering frequencies of resonators, Comm. Pure Appl. Math. 26 (1973), 549-563.

6. R. Brown, P. D. Hislop and A. Martinez, Eigenvalues and resonances for domains with tubes: Neumann boundary conditions, preprint.

7. I. Chavel and E. A. Feldman, Spectra of domains in compact manifolds, J. Funct. Anal. 30 (1978), 198-222. 
8. R. Courant and D. Hilbert, Methods of mathematical physics, Vol. I, Wiley-Interscience, New York, 1953.

9. K. Fukaya, Collapsing of Riemannian manifolds and eigenvalues of Laplace operator, Invent. Math. 87 (1987), 517-547.

10. D. Gilbarg and N. Trudinger, Elliptic partial differential equations of second order, SpringerVerlag, New York and Berlin, 1977.

11. J. K. Hale and G. Raugel, Reaction-diffusion equations on thin domains, J. Math. Pures Appl. 71 (1992), 33-95.

12. J. K. Hale and J. M. Vegas, A nonlinear parabolic equation with varying domain, Arch. Rational Mech. Anal. 86 (1984), 99-123.

13. R. Hempel, L. Seco and B. Simon, The essential spectrum of Neumann Laplacians on some bounded singular domains, J. Funct. Anal. 102 (1991), 448-483.

14. P. D. Hislop and A. Martinez, Scattering resonances of a Helmholtz resonator, Indiana Univ. Math. J. 40 (1991), 767-788.

15. S. Jimbo, The singularly perturbed domain and the characterization for the eigenfunctions with Neumann boundary conditions, J. Differential Equations 77 (1989), 322-350.

16. __ Perturbation formula of eigenvalues in a singularly perturbed domain, J. Math. Soc. Japan (2) 45 (1993), 339-356.

17. S. Jimbo and Y. Morita, Remarks on the behavior of certain eigenvalues on a singularly perturbed domain with several thin channels, Comm. Partial Differential Equations 17 (1992), 523-552.

18. M. Lobo-Hidalgo and E. Sanchez-Palencia, Sur certaines propriétés spectrales des perturbations du domaine dans les problémes aux limites, Comm. Partial Differential Equations 4 (1979), 1085-1098.

19. S. Ozawa, Singular variations of domains and eigenvalues of the Laplacian, Duke Math. J. 48 (1981), 769-778.

20. __ Spectra of domains with small spherical Neumann boundary, J. Fac. Sci. Univ. Tokyo 30 (1983), 259-277.

21. J. Rauch and M. Taylor, Potential and scattering theory on wildly perturbed domains, J. Funct. Anal. 18 (1975), 27-59.

22. J. M. Vegas, Bifurcation caused by perturbing the domain in an elliptic equation, J. Differential Equations 48 (1983), 189-226.

Center for Dynamical Systems and Nonlinear Studies, School of Mathematics, Georgia Institute of Technology, Atlanta, Georgia 30332 78712

Current address: Department of Mathematics, The University of Texas at Austin, Austin, Texas

E-mail address: arrieta@math.utexas.edu 